\title{
Meta-analysis identifies 13 new loci associated with waist-hip ratio and reveals sexual dimorphism in the genetic basis of fat
} distribution

Iris M Heid ${ }^{1,2,214}$, Anne U Jackson ${ }^{3,214}$, Joshua C Randall ${ }^{4,214}$, Thomas W Winkler ${ }^{1,214}$, Lu $Q^{5}{ }^{5,6,214}$, Valgerdur Steinthorsdottir ${ }^{7,214}$, Gudmar Thorleifsson ${ }^{7,214}$, M Carola Zillikens $^{8,9}$, Elizabeth K Speliotes ${ }^{10,11}$, Reedik Mägi ${ }^{4}$, Tsegaselassie Workalemahu ${ }^{5}$, Charles C White ${ }^{12}$, Nabila Bouatia-Naji ${ }^{13,14}$, Tamara B Harris ${ }^{15}$, Sonja I Berndt ${ }^{16}$, Erik Ingelsson ${ }^{17}$, Cristen $\mathbf{J}$ Willer $^{3}$, Michael N Weedon ${ }^{18}$, Jian'An Luan ${ }^{19}$, Sailaja Vedantam ${ }^{10,20}$, Tõnu Esko ${ }^{21,23}$ Tuomas O Kilpeläinen ${ }^{19}$, Zoltán Kutalik ${ }^{24,25}$, Shengxu Li ${ }^{19}$, Keri L Monda ${ }^{26}$, Anna L Dixon $^{27}$, Christopher C Holmes ${ }^{28,29}$, Lee M Kaplan ${ }^{11,30,31}$, Liming Liang ${ }^{32,33}$, Josine L Min $^{34}$, Miriam F Moffatt ${ }^{35}$, Cliona Molony ${ }^{36}$, George Nicholson ${ }^{29}$, Eric E Schadt ${ }^{37,38}$, Krina T Zondervan ${ }^{39}$, Mary F Feitosa ${ }^{40}$, Teresa Ferreira ${ }^{4}$, Hana Lango Allen ${ }^{18}$, Robert J Weyant ${ }^{3}$, Eleanor Wheeler ${ }^{41}$, Andrew R Wood ${ }^{18}$, MAGIC ${ }^{42}$, Karol Estrada ${ }^{8,9,43}$, Michael E Goddard $^{44,45}$, Guillaume Lettre ${ }^{46,47}$, Massimo Mangino ${ }^{48}$, Dale R Nyholt ${ }^{49}$, Shaun Purcell $^{50,52}$, Albert Vernon Smith ${ }^{53,54}$, Peter M Visscher ${ }^{55}$, Jian Yang ${ }^{55}$, Steven A McCarroll $50,56,57$, James Nemesh ${ }^{56}$, Benjamin F Voight ${ }^{50,56,57}$, Devin Absher ${ }^{58}$, Najaf Amin $^{43}$, Thor Aspelund ${ }^{53,54}$, Lachlan Coin ${ }^{59}$, Nicole L Glazer 60,61 , Caroline Hayward ${ }^{62}$, Nancy L Heard-costa ${ }^{63}$, Jouke-Jan Hottenga ${ }^{64}$, Åsa Johansson ${ }^{65,66}$, Toby Johnson 24,25,67,68, Marika Kaakinen 69,70 , Karen Kapur ${ }^{24,25}$, Shamika Ketkar ${ }^{40}$, Joshua W Knowles $^{71}$, Peter Kraft ${ }^{32,33}$, Aldi T Kraja ${ }^{40}$, Claudia Lamina ${ }^{2,72}$, Michael F Leitzmann ${ }^{1}$, Barbara McKnight ${ }^{73}$, Andrew P Morris ${ }^{4}$, Ken K Ong ${ }^{19}$, John R B Perry ${ }^{18}$, Marjolein J Peters $^{8,9}$, Ozren Polasek ${ }^{74,75}$, Inga Prokopenko 4,76 , Nigel W Rayner ${ }^{4,76}$, Samuli Ripatti ${ }^{77,78}$, Fernando Rivadeneira ${ }^{8,9,43}$, Neil R Robertson ${ }^{4,76}$, Serena Sanna ${ }^{79}$, Ulla Sovio ${ }^{59}$, Ida Surakka ${ }^{77,78}$, Alexander Teumer ${ }^{80}$, Sophie van Wingerden ${ }^{43}$, Veronique Vitart ${ }^{62}$, Jing Hua Zhao $^{19}$, Christine Cavalcanti-Proença ${ }^{13,14}$, Peter S Chines ${ }^{81}$, Eva Fisher ${ }^{82}$, Jennifer R Kulzer ${ }^{83}$, Cecile Lecoeur ${ }^{13,14}$, Narisu Narisu ${ }^{81}$, Camilla Sandholt ${ }^{84}$, Laura J Scott ${ }^{3}$, Kaisa Silander ${ }^{77,78}$, Klaus Stark ${ }^{85}$, Mari-Liis Tammesoo ${ }^{21}$, Tanya M Teslovich ${ }^{3}$, Nicholas John Timpson ${ }^{86}$, Richard M Watanabe ${ }^{87,88}$, Ryan Welch ${ }^{3}$, Daniel I Chasman ${ }^{30,89}$, Matthew N Cooper $^{90}$, John-Olov Jansson ${ }^{91}$, Johannes Kettunen ${ }^{77,78}$, Robert W Lawrence ${ }^{90}$, Niina Pellikka ${ }^{77,78}$, Markus Perola ${ }^{77,78}$, Liesbeth Vandenput ${ }^{92}$, Helene Alavere ${ }^{21}$, Peter Almgren $^{93}$, Larry D Atwood ${ }^{63}$, Amanda J Bennett ${ }^{76}$, Reiner Biffar ${ }^{94}$, Lori L Bonnycastle ${ }^{81}$, Stefan R Bornstein ${ }^{95}$, Thomas A Buchanan ${ }^{87,96}$, Harry Campbell ${ }^{97}$, Ian N M Day ${ }^{86}$, Mariano Dei $^{79}$, Marcus Dörr ${ }^{98}$, Paul Elliott ${ }^{59,99}$, Michael R Erdos ${ }^{81}$, Johan G Eriksson ${ }^{100,104}$, Nelson B Freimer ${ }^{105}$, Mao Fu ${ }^{106}$, Stefan Gaget ${ }^{13,14}$, Eco J C Geus ${ }^{64}$, Anette P Gjesing ${ }^{84}$, Harald Grallert $^{2}$, Jürgen Gräßler ${ }^{107}$, Christopher J Groves ${ }^{76}$, Candace Guiducci ${ }^{10}$, Anna-Liisa Hartikainen ${ }^{108}$, Neelam Hassanali ${ }^{76}$, Aki S Havulinna ${ }^{109}$, Karl-Heinz Herzig ${ }^{70,110,111}$, Andrew A Hicks ${ }^{112}$, Jennie Hui $90,113,114$, Wilmar Igl ${ }^{65}$, Pekka Jousilahti ${ }^{109}$, Antti Jula ${ }^{115}$, Eero Kajantie ${ }^{101,116}$, Leena Kinnunen ${ }^{117}$, Ivana Kolcic ${ }^{74}$, Seppo Koskinen ${ }^{109}$, Peter Kovacs $^{118}$, Heyo K Kroemer ${ }^{119}$, Vjekoslav Krzelj ${ }^{120}$, Johanna Kuusisto ${ }^{121}$, Kirsti Kvaloy ${ }^{122}$, Jaana Laitinen ${ }^{123}$, Olivier Lantieri ${ }^{124}$, G Mark Lathrop ${ }^{125}$, Marja-Liisa Lokki ${ }^{126}$, Robert N Luben $^{127}$, Barbara Ludwig ${ }^{95}$, Wendy L McArdle ${ }^{128}$, Anne McCarthy ${ }^{129}$, Mario A Morken ${ }^{81}$, Mari Nelis ${ }^{21,23}$, Matt J Neville ${ }^{76}$, Guillaume Paré ${ }^{130}$, Alex N Parker ${ }^{131}$, John F Peden ${ }^{4,132}$, Irene Pichler ${ }^{112}$, Kirsi H Pietiläinen ${ }^{133,134}$, Carl G P Platou 122,135, Anneli Pouta108,136, Martin Ridderstråle $^{137}$, Nilesh J Samani ${ }^{138,139}$, Jouko Saramies ${ }^{140}$, Juha Sinisalo ${ }^{141}$, Jan H Smit $^{142}$, Rona J Strawbridge ${ }^{143}$, Heather M Stringham ${ }^{3}$, Amy J Swift ${ }^{81}$, Maris TederLaving $^{22,23}$, Brian Thomson ${ }^{10}$, Gianluca Usala ${ }^{79}$, Joyce B J van Meurs ${ }^{8,9,43}$, Gert-Jan van Ommen $^{144,145}$, Vincent Vatin ${ }^{13,14}$, Claudia B Volpato ${ }^{112}$, Henri Wallaschofski ${ }^{146}$, G Bragi 
Walters $^{7}$, Elisabeth Widen ${ }^{77}$, Sarah H Wild ${ }^{97}$, Gonneke Willemsen ${ }^{64}$, Daniel R Witte ${ }^{147}$, Lina Zgaga $^{74}$, Paavo Zitting ${ }^{148}$, John P Beilby ${ }^{113,114,149}$, Alan L James ${ }^{114,150}$, Mika Kähönen ${ }^{151}$, Terho Lehtimäki ${ }^{152}$, Markku S Nieminen ${ }^{141}$, Claes Ohlsson ${ }^{92}$, Lyle J Palmer ${ }^{90,114}$, Olli Raitakari ${ }^{153,154}$, Paul M Ridker ${ }^{30,89}$, Michael Stumvoll ${ }^{155,156}$, Anke Tönjes ${ }^{155,157}$, Jorma Viikari ${ }^{158}$, Beverley Balkau ${ }^{159,160}$, Yoav Ben-Shlomo ${ }^{161}$, Richard N Bergman 87 , Heiner Boeing $^{82}$, George Davey Smith ${ }^{86}$, Shah Ebrahim ${ }^{162,163}$, Philippe Froguel ${ }^{13,14,164}$, Torben Hansen ${ }^{84,165}$, Christian Hengstenberg ${ }^{166,167}$, Kristian Hveem ${ }^{122}$, Bo Isomaa ${ }^{103,168}$, Torben Jørgensen ${ }^{169,170}$, Fredrik Karpe ${ }^{76,171}$, Kay-Tee Khaw ${ }^{127}$, Markku Laakso ${ }^{121}$, Debbie A Lawlor $^{86}$, Michel Marre ${ }^{172,173}$, Thomas Meitinger ${ }^{174,175}$, Andres Metspalu ${ }^{21,23}$, Kristian Midthjell $^{122}$, Oluf Pedersen ${ }^{84,176,177}$, Veikko Salomaa ${ }^{109}$, Peter E H Schwarz ${ }^{178}$, Tiinamaija Tuomi ${ }^{103,179,180}$, Jaakko Tuomilehto ${ }^{117,181,182}$, Timo T Valle ${ }^{117}$, Nicholas J Wareham ${ }^{19}$, Alice M Arnold ${ }^{73,183}$, Jacques S Beckmann ${ }^{24,184}$, Sven Bergmann ${ }^{24,25}$, Eric Boerwinkle ${ }^{185}$, Dorret I Boomsma ${ }^{64}$, Mark J Caulfield ${ }^{68}$, Francis S Collins ${ }^{81}$, Gudny Eiriksdottir ${ }^{53}$, Vilmundur Gudnason ${ }^{53,54}$, Ulf Gyllensten ${ }^{65}$, Anders Hamsten ${ }^{143}$, Andrew T Hattersley ${ }^{18}$, Albert Hofman ${ }^{9,43}$, Frank B Hu${ }^{5,6,32}$, Thomas Illig ${ }^{2}$, Carlos Iribarren ${ }^{186,187}$, Marjo-Riitta Jarvelin ${ }^{59,69,70,136}$, W H Linda Kao ${ }^{188}$, Jaakko Kaprio ${ }^{77,133,189}$, Lenore J Launer ${ }^{15}$, Patricia B Munroe $^{68}$, Ben Oostra ${ }^{190}$, Brenda W Penninx ${ }^{142,191,192}$, Peter P Pramstaller ${ }^{112,193,194}$, Bruce M Psaty ${ }^{195,196}$, Thomas Quertermous ${ }^{71}$, Aila Rissanen ${ }^{134}$, Igor Rudan ${ }^{97,120}$, Alan R Shuldiner $^{106,197}$, Nicole Soranzo ${ }^{41,48}$, Timothy D Spector ${ }^{48}$, Ann-Christine Syvanen ${ }^{198}$, Manuela Uda ${ }^{79}$, André Uitterlinden $8,9,43$, Henry Völzke ${ }^{199}$, Peter Vollenweider ${ }^{200}$, James F Wilson $^{97}$, Jacqueline C Witteman ${ }^{9,43}$, Alan F Wright ${ }^{62}$, Gonçalo R Abecasis ${ }^{3}$, Michael Boehnke $^{3}$, Ingrid B Borecki ${ }^{40,201}$, Panos Deloukas ${ }^{41}$, Timothy M Frayling ${ }^{18}$, Leif C Groop ${ }^{93}$, Talin Haritunians ${ }^{202}$, David J Hunter 5,6,32, Robert C Kaplan ${ }^{203}$, Kari E North ${ }^{26,204}$, Jeffrey R O'connell ${ }^{106}$, Leena Peltonen 41,51,77,101,205, David Schlessinger ${ }^{206}$, David P Strachan ${ }^{207}$, Joel N Hirschhorn 10,20,208, Themistocles L Assimes ${ }^{71}$, H-Erich Wichmann 2,209,210, Unnur Thorsteinsdottir $^{7,211}$, Cornelia M van Duijn ${ }^{9,43}$, Kari Stefansson $7,211,215$, L Adrienne Cupples $^{12,215}$, Ruth J F Loos ${ }^{19,215}$, Inês Barroso ${ }^{41,212,215}$, Mark I McCarthy 4,76,171,215, Caroline S Fox ${ }^{213,215}$, Karen L Mohlke ${ }^{83,215}$, and Cecilia M Lindgren $4,76,215$

${ }^{1}$ Regensburg University Medical Center, Department of Epidemiology and Preventive Medicine, Regensburg, Germany. ${ }^{2}$ Institute of Epidemiology, Helmholtz Zentrum München-German Research Center for Environmental Health, Neuherberg, Germany. ${ }^{3}$ Department of Biostatistics, Center for Statistical Genetics, University of Michigan, Ann Arbor, Michigan, USA. ${ }^{4}$ Wellcome Trust Centre for Human Genetics, University of Oxford, Oxford, UK. ${ }^{5}$ Department of Nutrition, Harvard School of Public Health, Boston, Massachusetts, USA. ${ }^{6}$ Channing Laboratory, Department of Medicine, Brigham and Women's Hospital and Harvard Medical School, Boston, Massachusetts, USA. ${ }^{7}$ deCODE Genetics, Reykjavik, Iceland. ${ }^{8}$ Department of Internal Medicine, Erasmus Medical Center (MC), Rotterdam, The Netherlands. ${ }^{9}$ Netherlands Genomics Initiative (NGl)-sponsored Netherlands Consortium for Healthy Aging (NCHA), Rotterdam, The Netherlands. ${ }^{10}$ Metabolism Initiative and Program in Medical and Population Genetics, Broad Institute, Cambridge, Massachusetts, USA. ${ }^{11}$ Division of Gastroenterology, Massachusetts General Hospital, Boston, Massachusetts, USA. ${ }^{12}$ Department of Biostatistics, Boston University School of Public Health, Boston, Massachusetts, USA. ${ }^{13}$ Centre National de la Recherche Scientifique (CNRS), UMR8199-IBL-Institut Pasteur de Lille, Lille, France. ${ }^{14}$ University Lille Nord de France, Lille, France. ${ }^{15}$ Laboratory of Epidemiology, Demography, Biometry, National Institute on Aging, National Institutes of Health, Bethesda, Maryland, USA. ${ }^{16}$ Division of Cancer

Epidemiology and Genetics, National Cancer Institute, National Institutes of Health, Department of Health and Human Services, Bethesda, Maryland, USA. ${ }^{17}$ Department of Medical

Epidemiology and Biostatistics, Karolinska Institutet, Stockholm, Sweden. ${ }^{18}$ Genetics of Complex Traits, Peninsula College of Medicine and Dentistry, University of Exeter, Exeter, UK. ${ }^{19}$ Medical Research Council (MRC) Epidemiology Unit, Institute of Metabolic Science, Addenbrooke's Hospital, Cambridge, UK. ${ }^{20}$ Divisions of Genetics and Endocrinology and Program in Genomics, 
Children's Hospital, Boston, Massachusetts, USA. ${ }^{21}$ Estonian Genome Center, University of Tartu, Tartu, Estonia. ${ }^{22}$ Estonian Biocenter, Tartu, Estonia. ${ }^{23}$ Institute of Molecular and Cell Biology, University of Tartu, Tartu, Estonia. ${ }^{24}$ Department of Medical Genetics, University of Lausanne, Lausanne, Switzerland. ${ }^{25}$ Swiss Institute of Bioinformatics, Lausanne, Switzerland. ${ }^{26}$ Department of Epidemiology, School of Public Health, University of North Carolina at Chapel Hill, Chapel Hill, North Carolina, USA. ${ }^{27}$ Department of Pharmacy and Pharmacology, University of Bath, Bath, UK. ${ }^{28} \mathrm{MRC}$ Harwell, Harwell Science and Innovation Campus, Oxfordshire, UK. ${ }^{29}$ Department of Statistics, University of Oxford, Oxford, UK. ${ }^{30}$ Harvard Medical School, Boston, Massachusetts, USA. ${ }^{31}$ Massachusetts General Hospital (MGH) Weight Center, Massachusetts General Hospital, Boston, Massachusetts, USA. ${ }^{32}$ Department of Epidemiology, Harvard School of Public Health, Boston, Massachusetts, USA. ${ }^{33}$ Department of Biostatistics, Harvard School of Public Health, Boston, Massachusetts, USA. ${ }^{34}$ Human Genetics, Leiden University Medical Center, Leiden, The Netherlands. ${ }^{35}$ National Heart and Lung Institute, Imperial College London, London, UK. ${ }^{36}$ Merck Research Laboratories, Merck \& Co., Inc., Boston, Massachusetts, USA. ${ }^{37}$ Pacific Biosciences, Menlo Park, California, USA. ${ }^{38}$ Sage Bionetworks, Seattle, Washington, USA. ${ }^{39}$ Genetic and Genomic Epidemiology Unit, Wellcome Trust Centre for Human Genetics, Oxford, UK. ${ }^{40}$ Department of Genetics, Washington University School of Medicine, St. Louis, Missouri, USA. ${ }^{41}$ Wellcome Trust Sanger Institute, Hinxton, Cambridge, UK. ${ }^{42}$ On behalf of the MAGIC (Meta-Analyses of Glucose and Insulin-related traits Consortium) investigators.

${ }^{43}$ Department of Epidemiology, Erasmus MC, Rotterdam, The Netherlands. ${ }^{44}$ University of Melbourne, Parkville, Australia. ${ }^{45}$ Department of Primary Industries, Melbourne, Victoria, Australia. ${ }^{46}$ Montreal Heart Institute, Montreal, Quebec, Canada. ${ }^{47}$ Department of Medicine, Université de Montréal, Montreal, Quebec, Canada. ${ }^{48}$ Department of Twin Research and Genetic Epidemiology, King's College London, London, UK. ${ }^{49}$ Neurogenetics Laboratory, Queensland Institute of Medical Research, Queensland, Australia. ${ }^{50}$ Center for Human Genetic Research, Massachusetts General Hospital, Boston, Massachusetts, USA. ${ }^{51}$ The Broad Institute of Harvard and Massachusetts Institute of Technology (MIT), Cambridge, Massachusetts, USA.

${ }^{52}$ Department of Psychiatry, Harvard Medical School, Boston, Massachusetts, USA. ${ }^{53}$ Icelandic Heart Association, Kopavogur, Iceland. ${ }^{54}$ University of Iceland, Reykjavik, Iceland. ${ }^{55}$ Queensland Statistical Genetics Laboratory, Queensland Institute of Medical Research, Queensland, Australia. ${ }^{56}$ Program in Medical and Population Genetics, Broad Institute of Harvard and MIT, Cambridge, Massachusetts, USA. ${ }^{57}$ Department of Molecular Biology, Massachusetts General Hospital, Boston, Massachusetts, USA. ${ }^{58}$ Hudson Alpha Institute for Biotechnology, Huntsville, Alabama, USA. ${ }^{59}$ Department of Epidemiology and Biostatistics, School of Public Health, Faculty of Medicine, Imperial College London, London, UK. ${ }^{60}$ Department of Medicine, University of Washington, Seattle, Washington, USA. ${ }^{61}$ Cardiovascular Health Research Unit, University of Washington, Seattle, Washington, USA. ${ }^{62} \mathrm{MRC}$ Human Genetics Unit, Institute for Genetics and Molecular Medicine, Western General Hospital, Edinburgh, Scotland, UK. ${ }^{63}$ Department of Neurology, Boston University School of Medicine, Boston, Massachusetts, USA. ${ }^{64}$ Department of Biological Psychology, Vrije Universiteit (VU) University Amsterdam, Amsterdam, The Netherlands. ${ }^{65}$ Department of Genetics and Pathology, Rudbeck Laboratory, University of Uppsala, Uppsala, Sweden. ${ }^{66}$ Department of Cancer Research and Molecular Medicine, Faculty of Medicine, Norwegian University of Science and Technology (NTNU), Trondheim, Norway. ${ }^{67}$ Clinical Pharmacology, William Harvey Research Institute, Barts and The London School of Medicine and Dentistry, Queen Mary, University of London, London, UK. ${ }^{68} \mathrm{Clinical}$ Pharmacology and Barts and The London Genome Centre, William Harvey Research Institute, Barts and The London School of Medicine and Dentistry, Queen Mary University of London, London UK. ${ }^{69}$ Institute of Health Sciences, University of Oulu, Oulu, Finland. ${ }^{70}$ Biocenter Oulu, University of Oulu, Oulu, Finland. ${ }^{71}$ Department of Medicine, Stanford University School of Medicine, Stanford, California, USA. ${ }^{72}$ Division of Genetic Epidemiology, Department of Medical Genetics, Molecular and Clinical Pharmacology, Innsbruck Medical University, Innsbruck, Austria. ${ }^{73}$ Department of 
Biostatistics, University of Washington, Seattle, Washington, USA. ${ }^{74}$ Andrija Stampar School of Public Health, Medical School, University of Zagreb, Zagreb, Croatia. ${ }^{75}$ Gen-Info Ltd, Zagreb, Croatia. ${ }^{76}$ Oxford Centre for Diabetes, Endocrinology and Metabolism, University of Oxford, Oxford, UK. ${ }^{77}$ Institute for Molecular Medicine Finland (FIMM), University of Helsinki, Helsinki, Finland. ${ }^{78}$ National Institute for Health and Welfare, Department of Chronic Disease Prevention, Unit of Public Health Genomics, Helsinki, Finland. ${ }^{79}$ Istituto di Neurogenetica e

Neurofarmacologia del CNR, Monserrato, Cagliari, Italy. ${ }^{80}$ Interfaculty Institute for Genetics and Functional Genomics, Ernst-Moritz-Arndt-University Greifswald, Greifswald, Germany. ${ }^{81}$ National Human Genome Research Institute, National Institutes of Health, Bethesda, Maryland, USA. ${ }^{82}$ Department of Epidemiology, German Institute of Human Nutrition Potsdam-Rehbruecke, Nuthetal, Germany. ${ }^{83}$ Department of Genetics, University of North Carolina, Chapel Hill, North Carolina, USA. ${ }^{84}$ Hagedorn Research Institute, Gentofte, Denmark. ${ }^{85}$ Regensburg University Medical Center, Clinic and Policlinic for Internal Medicine II, Regensburg, Germany. ${ }^{86} \mathrm{MRC}$ Centre for Causal Analyses in Translational Epidemiology, Department of Social Medicine, Oakfield House, Bristol, UK. ${ }^{87}$ Department of Physiology and Biophysics, Keck School of Medicine, University of Southern California, Los Angeles, California, USA. ${ }^{88}$ Department of Preventive Medicine, Keck School of Medicine, University of Southern California, Los Angeles, California, USA. ${ }^{89}$ Division of Preventive Medicine, Brigham and Women's Hospital, Boston, Massachusetts, USA. ${ }^{90}$ Centre for Genetic Epidemiology and Biostatistics, University of Western Australia, Crawley, Western Australia, Australia. ${ }^{91}$ Department of Physiology, Institute of Neuroscience and Physiology, Sahlgrenska Academy, University of Gothenburg, Gothenburg, Sweden. ${ }^{92}$ Department of Internal Medicine, Institute of Medicine, Sahlgrenska Academy, University of Gothenburg, Gothenburg, Sweden. ${ }^{93}$ Lund University Diabetes Centre, Department of Clinical Sciences, Lund University, Malmö, Sweden. ${ }^{94}$ Zentrum für Zahn-, Mund- und Kieferheilkunde, Greifswald, Germany. ${ }^{95}$ Department of Medicine III, University of Dresden, Dresden, Germany. ${ }^{96}$ Division of Endocrinology, Keck School of Medicine, University of Southern California, Los Angeles, California, USA. ${ }^{97}$ Centre for Population Health Sciences, University of Edinburgh, Teviot Place, Edinburgh, Scotland, UK. ${ }^{98}$ Department of Internal Medicine B, ErnstMoritz-Arndt University, Greifswald, Germany. ${ }^{99}$ MRC-Health Protection Agency (HPA) Centre for Environment and Health, London, UK. ${ }^{100}$ Department of General Practice and Primary Health Care, University of Helsinki, Helsinki, Finland. ${ }^{101}$ National Institute for Health and Welfare, Helsinki, Finland. ${ }^{102} \mathrm{Helsinki}$ University Central Hospital, Unit of General Practice, Helsinki, Finland. ${ }^{103}$ Folkhalsan Research Centre, Helsinki, Finland. ${ }^{104}$ Vasa Central Hospital, Vasa, Finland. ${ }^{105}$ Center for Neurobehavioral Genetics, University of California, Los Angeles, California, USA. ${ }^{106}$ Department of Medicine, University of Maryland School of Medicine, Baltimore, Maryland, USA. ${ }^{107}$ Department of Medicine III, Pathobiochemistry, University of Dresden, Dresden, Germany. ${ }^{108}$ Department of Clinical Sciences/Obstetrics and Gynecology, University of Oulu, Oulu, Finland. ${ }^{109}$ National Institute for Health and Welfare, Department of Chronic Disease Prevention, Chronic Disease Epidemiology and Prevention Unit, Helsinki, Finland. ${ }^{110}$ Institute of Biomedicine, Department of Physiology, University of Oulu, Oulu, Finland. ${ }^{111}$ Department of Psychiatry, Kuopio University Hospital and University of Kuopio, Kuopio, Finland. ${ }^{112}$ Institute of Genetic Medicine, European Academy Bozen-Bolzano (EURAC), Bolzano-Bozen, Italy (affiliated Institute of the University of Lübeck, Lübeck, Germany). ${ }^{113}$ PathWest Laboratory of Western Australia, Department of Molecular Genetics, J Block, QEII Medical Centre, Nedlands, Western Australia, Australia. ${ }^{114}$ Busselton Population Medical Research Foundation Inc., Sir Charles Gairdner Hospital, Nedlands, Western Australia, Australia. ${ }^{115}$ National Institute for Health and Welfare, Department of Chronic Disease Prevention, Population Studies Unit, Turku, Finland. ${ }^{116}$ Hospital for Children and Adolescents, Helsinki University Central Hospital and University of Helsinki, Helsinki, Finland. ${ }^{117}$ National Institute for Health and Welfare, Diabetes Prevention Unit, Helsinki, Finland. ${ }^{118}$ Interdisciplinary Centre for Clinical Research, University of Leipzig, Leipzig, Germany. ${ }^{119}$ Institut für Pharmakologie, Universität Greifswald, Greifswald, Germany. ${ }^{120}$ Croatian 
Centre for Global Health, School of Medicine, University of Split, Split, Croatia. ${ }^{121}$ Department of Medicine, University of Kuopio and Kuopio University Hospital, Kuopio, Finland. ${ }^{122}$ NordTrøndelag Health Study (HUNT) Research Centre, Department of Public Health and General Practice, Norwegian University of Science and Technology, Levanger, Norway. ${ }^{123}$ Finnish Institute of Occupational Health, Oulu, Finland. ${ }^{124}$ Institut inter-regional pour la sante (IRSA), La Riche, France. ${ }^{125}$ Centre National de Genotypage, Evry, Paris, France. ${ }^{126}$ Transplantation Laboratory, Haartman Institute, University of Helsinki, Helsinki, Finland. ${ }^{127}$ Department of Public Health and Primary Care, Institute of Public Health, University of Cambridge, Cambridge, UK. ${ }^{128}$ Avon Longitudinal Study of Parents and Children (ALSPAC) Laboratory, Department of Social Medicine, University of Bristol, Bristol, UK. ${ }^{129}$ Division of Health, Research Board, An Bord Taighde Sláinte, Dublin, Ireland. ${ }^{130}$ Department of Pathology and Molecular Medicine, McMaster University, Hamilton, Ontario, Canada. ${ }^{131}$ Amgen, Cambridge, Massachusetts, USA.

${ }^{132}$ Department of Cardiovascular Medicine, University of Oxford, John Radcliffe Hospital, Headington, Oxford, UK. ${ }^{133}$ Finnish Twin Cohort Study, Department of Public Health, University of Helsinki, Helsinki, Finland. ${ }^{134}$ Obesity Research Unit, Department of Psychiatry, Helsinki University Central Hospital, Helsinki, Finland. ${ }^{135}$ Department of Medicine, Levanger Hospital, The Nord-Trøndelag Health Trust, Levanger, Norway. ${ }^{136}$ National Institute for Health and Welfare, Oulu, Finland. ${ }^{137}$ Department of Clinical Sciences, Lund University, Malmö, Sweden.

${ }^{138}$ Department of Cardiovascular Sciences, University of Leicester, Glenfield Hospital, Leicester, UK. ${ }^{139}$ Leicester National Institute for Health Research (NIHR) Biomedical Research Unit in Cardiovascular Disease, Glenfield Hospital, Leicester, UK. ${ }^{140}$ South Karelia Central Hospital, Lappeenranta, Finland. ${ }^{141}$ Division of Cardiology, Cardiovascular Laboratory, Helsinki University Central Hospital, Helsinki, Finland. ${ }^{142}$ Department of Psychiatry/Instituut voor Extramuraal Geneeskundig Onderzoek (EMGO) Institute, VU University Medical Center, Amsterdam, The Netherlands. ${ }^{143}$ Atherosclerosis Research Unit, Department of Medicine, Solna, Karolinska Institutet, Karolinska University Hospital, Stockholm, Sweden. ${ }^{144}$ Department of Human Genetics, Leiden University Medical Center, Leiden, The Netherlands. ${ }^{145}$ Center of Medical Systems Biology, Leiden University Medical Center, Leiden, The Netherlands. ${ }^{146}$ Institut für Klinische Chemie und Laboratoriumsmedizin, Universität Greifswald, Greifswald, Germany. ${ }^{147}$ Steno Diabetes Center, Gentofte, Denmark. ${ }^{148}$ Department of Physiatrics, Lapland Central Hospital, Rovaniemi, Finland. ${ }^{149}$ School of Pathology and Laboratory Medicine, University of Western Australia, Nedlands, Western Australia, Australia. ${ }^{150}$ School of Medicine and Pharmacology, University of Western Australia, Perth, Western Australia, Australia. ${ }^{151}$ Department of Clinical Physiology, University of Tampere and Tampere University Hospital, Tampere, Finland. ${ }^{152}$ Department of Clinical Chemistry, University of Tampere and Tampere University Hospital, Tampere, Finland. ${ }^{153}$ Research Centre of Applied and Preventive Cardiovascular Medicine, University of Turku, Turku, Finland. ${ }^{154}$ The Department of Clinical Physiology, Turku University Hospital, Turku, Finland. ${ }^{155}$ Department of Medicine, University of Leipzig, Leipzig, Germany. ${ }^{156}$ Leipziger Interdisziplinärer Forschungskomplex zu molekularen Ursachen umwelt- und lebensstilassoziierter Erkrankungen (LIFE) Study Centre, University of Leipzig, Leipzig, Germany. ${ }^{157}$ Coordination Centre for Clinical Trials, University of Leipzig, Leipzig, Germany. ${ }^{158}$ Department of Medicine, University of Turku and Turku University Hospital, Turku, Finland. ${ }^{159}$ INSERM Centre de Recherche en Epidémiologie et Santé des Populations (CESP) U1018, Villejuif, France. ${ }^{160}$ University Paris Sud 11, Unité Mixte de Recherche en Santé (UMRS) 1018, Villejuif, France. ${ }^{161}$ Department of Social Medicine, University of Bristol, Bristol, UK. ${ }^{162}$ The London School of Hygiene and Tropical Medicine, London, UK. ${ }^{163}$ South Asia Network for Chronic Disease, New Delhi, India. ${ }^{164}$ Department of Genomics of Common Disease, School of Public Health, Imperial College London, London, UK. ${ }^{165}$ Faculty of Health Science, University of Southern Denmark, Odense, Denmark. ${ }^{166} \mathrm{Klinik}$ und Poliklinik für Innere Medizin II, Universität Regensburg, Regensburg, Germany. ${ }^{167}$ Regensburg University Medical Center, Innere Medizin II, Regensburg, Germany. ${ }^{168}$ Department of Social Services and Health Care, Jakobstad, Finland. ${ }^{169}$ Research 
Centre for Prevention and Health, Glostrup University Hospital, Glostrup, Denmark. ${ }^{170}$ Faculty of Health Science, University of Copenhagen, Copenhagen, Denmark. ${ }^{171}$ NIHR Oxford Biomedical Research Centre, Churchill Hospital, Oxford, UK. ${ }^{172}$ Department of Endocrinology, Diabetology and Nutrition, Bichat-Claude Bernard University Hospital, Assistance Publique des Hôpitaux de Paris, Paris, France. ${ }^{173}$ Cardiovascular Genetics Research Unit, Université Henri Poincaré-Nancy 1, Nancy, France. ${ }^{174}$ Institute of Human Genetics, Klinikum rechts der Isar der Technischen Universität München, Munich, Germany. ${ }^{175}$ Institute of Human Genetics, Helmholtz Zentrum München-German Research Center for Environmental Health, Neuherberg, Germany. ${ }^{176}$ Institute of Biomedical Sciences, University of Copenhagen, Copenhagen, Denmark. ${ }^{177}$ Faculty of Health Science, University of Aarhus, Aarhus, Denmark. ${ }^{178}$ Department of Medicine III, Prevention and Care of Diabetes, University of Dresden, Dresden, Germany. ${ }^{179}$ Department of Medicine, Helsinki University Central Hospital, Helsinki, Finland. ${ }^{180}$ Research Program of Molecular Medicine, University of Helsinki, Helsinki, Finland. ${ }^{181} \mathrm{Hjelt}$ Institute, Department of Public Health, University of Helsinki, Helsinki, Finland. ${ }^{182}$ South Ostrobothnia Central Hospital, Seinajoki, Finland. ${ }^{183}$ Collaborative Health Studies Coordinating Center, Seattle, Washington, USA. ${ }^{184}$ Service of Medical Genetics, Centre Hospitalier Universitaire Vaudois (CHUV) University Hospital, Lausanne, Switzerland. ${ }^{185} \mathrm{Human}$ Genetics Center and Institute of Molecular Medicine, University of Texas Health Science Center, Houston, Texas, USA. ${ }^{186}$ Division of Research, Kaiser Permanente Northern California, Oakland, California, USA. ${ }^{187}$ Department of Epidemiology and Biostatistics, University of California, San Francisco, San Francisco, California, USA. ${ }^{188}$ Department of Epidemiology and Medicine, Johns Hopkins Bloomberg School of Public Health, Baltimore, Maryland, USA. ${ }^{189}$ National Institute for Health and Welfare, Department of Mental Health and Substance Abuse Services, Unit for Child and Adolescent Mental Health, Helsinki, Finland. ${ }^{190}$ Department of Clinical Genetics, Erasmus MC, Rotterdam, The Netherlands. ${ }^{191}$ Department of Psychiatry, Leiden University Medical Centre, Leiden, The Netherlands.

${ }^{192}$ Department of Psychiatry, University Medical Centre Groningen, Groningen, The Netherlands. ${ }^{193}$ Department of Neurology, General Central Hospital, Bolzano, Italy. ${ }^{194}$ Department of Neurology, University of Lübeck, Lübeck, Germany. ${ }^{195}$ Departments of Epidemiology, Medicine and Health Services, University of Washington, Seattle, Washington, USA. ${ }^{196}$ Group Health Research Institute, Group Health, Seattle, Washington, USA. ${ }^{197}$ Geriatrics Research and Education Clinical Center, Baltimore Veterans Administration Medical Center, Baltimore, Maryland, USA. ${ }^{198}$ Uppsala University, Department of Medical Sciences, Molecular Medicine, Uppsala, Sweden. ${ }^{199}$ Institut für Community Medicine, Greifswald, Germany. ${ }^{200}$ Department of Internal Medicine, Centre Hospitalier Universitaire Vaudois (CHUV) University Hospital, Lausanne, Switzerland. ${ }^{201}$ Division of Biostatistics, Washington University School of Medicine, St. Louis, Missouri, USA. ${ }^{202}$ Medical Genetics Institute, Cedars-Sinai Medical Center, Los Angeles, California, USA. ${ }^{203}$ Department of Epidemiology and Population Health, Albert Einstein College of Medicine, Bronx, New York, USA. ${ }^{204}$ Carolina Center for Genome Sciences, School of Public Health, University of North Carolina Chapel Hill, Chapel Hill, North Carolina, USA. ${ }^{205}$ Department of Medical Genetics, University of Helsinki, Helsinki, Finland. ${ }^{206}$ Laboratory of Genetics, National Institute on Aging, Baltimore, Maryland, USA. ${ }^{207}$ Division of Community Health Sciences, St George's, University of London, London, UK. ${ }^{208}$ Department of Genetics, Harvard Medical School, Boston, Massachusetts, USA. ${ }^{209}$ Klinikum Grosshadern, Munich, Germany. ${ }^{210}$ LudwigMaximilians-Universität, Institute of Medical Informatics, Biometry and Epidemiology, Munich, Germany. ${ }^{211}$ Faculty of Medicine, University of Iceland, Reykjavík, Iceland. ${ }^{212}$ University of Cambridge Metabolic Research Labs, Institute of Metabolic Science Addenbrooke's Hospital, Cambridge, UK. ${ }^{213}$ Division of Intramural Research, National Heart, Lung, and Blood Institute, Framingham Heart Study, Framingham, Massachusetts, USA.

\section{Abstract}


Waist-hip ratio (WHR) is a measure of body fat distribution and a predictor of metabolic consequences independent of overall adiposity. WHR is heritable, but few genetic variants influencing this trait have been identified. We conducted a meta-analysis of 32 genome-wide association studies for WHR adjusted for body mass index (comprising up to 77,167 participants), following up 16 loci in an additional 29 studies (comprising up to 113,636 subjects). We identified 13 new loci in or near RSPO3, VEGFA, TBX15-WARS2, NFE2L3, GRB14, DNM3-PIGC, ITPR2-SSPN, LY86, HOXC13, ADAMTS9, ZNRF3-KREMEN1, NISCH-STAB1 and CPEB4 (P $=1.9 \times 10^{-9}$ to $P=1.8 \times 10^{-40}$ ) and the known signal at $L Y P L A L 1$. Seven of these loci exhibited marked sexual dimorphism, all with a stronger effect on WHR in women than men ( $P$ for sex difference $=1.9 \times 10^{-3}$ to $P=1.2 \times 10^{-13}$ ). These findings provide evidence for multiple loci that modulate body fat distribution independent of overall adiposity and reveal strong gene-by-sex interactions.

Central obesity and body fat distribution, as measured by waist circumference and WHR, are associated with individual risk of type 2 diabetes (T2D) ${ }^{1},{ }^{2}$ and coronary heart disease ${ }^{3}$ and with mortality from all causes ${ }^{4}$. These effects are independent of overall adiposity as measured by body mass index (BMI). WHR is of particular interest as a measure of body fat distribution because it integrates the adverse metabolic risk associated with increasing waist circumference with the more protective role of gluteal fat deposition with respect to diabetes, hypertension and dyslipidemia ${ }^{5,}$.

There is abundant evidence that body fat distribution is influenced by genetic loci distinct from those regulating BMI and overall adiposity. First, even after accounting for BMI, individual variation in WHR is heritable 7,8 , with heritability estimates ranging from $22 \%$ $61 \%{ }^{7}-10$. Second, the striking abnormalities of regional fat deposition associated with lipodystrophic syndromes demonstrate that genetic variation can have dramatic effects on the development and maintenance of specific fat depots ${ }^{11},{ }^{12}$. Third, in a previous genomewide association analysis, we identified a locus near $L Y P L A L 1$ strongly associated with WHR independent of any effects on $\mathrm{BMI}^{13}$, providing proof of principle for the genetic control of body fat distribution distinct from that of overall adiposity.

Within the Genetic Investigation of Anthropometric Traits (GIANT) consortium, we performed a large-scale meta-analysis of genome-wide association studies (GWAS) informative for WHR using adjustment for BMI to focus discovery toward genetic loci associated with body fat distribution rather than overall adiposity ${ }^{14}{ }^{16}$.

\section{RESULTS}

\section{Genome-wide significant association of WHR with 14 SNPs}

We conducted a two-stage study among individuals of European descent (Supplementary Table 1 and Online Methods). In the discovery stage, up to 2,850,269 imputed and genotyped SNPs were examined in 32 GWAS comprising up to 77,167 participants informative for anthropometric measures of body fat distribution. We performed a fixedeffects meta-analysis of WHR, employing study-specific linear regression adjusted for BMI and age, stratified by gender, and using an additive genetic model. After genomic control adjustment per each individual study and in the meta-analysis, these analyses revealed a substantial excess of low $P$ values (Fig. 1a,b).

We selected SNPs representing the top 16 independent (defined as being located $>1 \mathrm{Mb}$ apart) regions of association (discovery $P<1.4 \times 10^{-6}$; Table 1 ) and evaluated them in 29 additional, independent studies (comprising up to 113,636 individuals) using a mixture of in silico data and de novo genotyping. In these follow-up studies, 14 of the 16 SNPs analyzed 
showed strong directionally consistent evidence for replication $\left(P<1.0 \times 10^{-3}\right)$ and ten SNPs reached genome-wide significance $\left(P<5.0 \times 10^{-8}\right)$. Joint analysis of the discovery and follow-up results revealed genome-wide significant associations for 14 signals (with $P$ values between $1.9 \times 10^{-9}$ and $1.8 \times 10^{-40}$; Table 1$)$. Between-study heterogeneity was low $\left(I^{2}<30 \%\right)$ for all but two signals (GRB14 and LYPLAL1; Supplementary Note), and all 14 associations remained genome-wide significant in a random-effects meta-analysis (Supplementary Table 2).

One of these SNPs, rs4846567, is in linkage disequilibrium (LD) $\left(I^{2}=0.64, D^{\prime}=0.84\right.$; HapMap European CEU population) with the previously reported WHR-associated variant near LYPLAL1 (rs2605100) ${ }^{13}$. The remaining 13 loci were in or near genes not previously associated with WHR or other measures of adiposity: RSPO3, VEGFA, TBX15-WARS2, NFE2L3, GRB14, DNM3-PIGC, ITPR2-SSPN, LY86, HOXC13, ADAMTS9, ZNRF3KREMEN1, NISCH-STAB1 and CPEB4 (Fig. 2). These 14 loci explain $1.03 \%$ of the variance in WHR (after adjustment for BMI, age and sex), with each locus contributing from $0.02 \%$ (ZNRF3-KREMENI) to $0.14 \%$ (RSPO3) of the variance based on effect estimates in the follow-up stage.

\section{Sexual dimorphism at several of the WHR loci}

Given the known sexual dimorphism of WHR and the evidence from variance decomposition studies that this reflects sex-specific genetic effects ${ }^{17}$, we performed sexspecific meta-analyses for the 14 WHR-associated SNPs. These analyses included up to 108,979 women ( 42,735 in the discovery stage and 66,244 in the follow up) and 82,483 men (34,601 in the discovery and 47,882 in the follow up). In a joint analysis of discovery and follow-up data, 12 of the 14 SNPs reached genome-wide significance in women, but only three SNPs reached genome-wide significance in men (Table 2). At all but one locus (TBX15-WARS2), effect-size estimates were numerically greater in women. At seven of the loci (those near RSPO3, VEGFA, GRB14, LYPLAL1, HOXC13, ITPR2-SSPN and $A D A M T S$ ), there were marked differences in sex-specific $\beta$ coefficients (with $P$ values ranging from $1.9 \times 10^{-3}$ to $1.2 \times 10^{-13}$ ). All loci displayed consistent patterns of sexspecific differences in both the discovery and follow-up studies (Table 2). These 14 loci explain $1.34 \%$ of the variance in WHR (after adjustment for BMI and age) in women but only $0.46 \%$ of the variance in WHR in men.

\section{Association with other anthropometric measures}

By focusing on WHR after adjustment for BMI, our goal was to detect effects on body fat distribution independent of those influencing overall adiposity. As expected, we found very little evidence that known BMI-associated variants were detected in our WHR analysis. Of the ten loci shown to be associated with BMI in previous $\mathrm{GWAS}^{14},{ }^{15},{ }^{18}$, only two showed nominally significant $(P<0.05)$ associations for BMI-adjusted WHR in the discovery analysis $\left(F T O, \mathrm{rs} 8050136, P=0.03, n=77,074 ;\right.$ TMEM18, rs6548238, $P=3.0 \times 10^{-3}, n=$ 77,016).

We also tested the 14 WHR-associated SNPs for their effect on BMI using data from up to 242,530 participants available from the GIANT consortium (including most of the studies available for WHR association). Of the 14 WHR loci, four (near TBX15-WARS2, CPEB4, LYPLAL1 and GRB14) also showed evidence of association with BMI $\left(4.1 \times 10^{-3} \leq P \leq\right.$ $3.2 \times 10^{-6}$ ), with the WHR-increasing allele associated with decreased BMI (Supplementary Table 3). After adding an interaction term of SNP with BMI into the model, we observed that BMI modified the WHR association at the LY86 locus $\left(P\right.$ for interaction $=9.5 \times 10^{-5}$ ), with a larger WHR effect among obese individuals compared to non-obese individuals (Supplementary Note). 
To determine whether the WHR-associated signals exert their effects primarily through an effect on waist or hip circumference, we performed meta-analyses for these specific phenotypes in the discovery and follow-up studies (Supplementary Tables 1 and 3). Overall, we observed stronger associations for hip circumference than for waist circumference. Effect-size estimates were numerically greater for hip circumference than for waist circumference at 11 of the 14 loci, and there were nominal associations $(P<0.05)$ with hip circumference for 12 of the WHR-associated loci but there were only four associations with waist circumference. In both sexes, the WHR-associated loci displaying nominal association with hip circumference always featured the WHR-increasing allele associated with reduced hip circumference. In contrast, we observed sexual dimorphism in the pattern of waist circumference associations. In women, the WHR-increasing allele at all 14 loci was associated with increased waist circumference, whereas this was only true for six of these loci in men (Fig. 3). At GRB14, for example, the WHR-increasing allele was associated with increased waist circumference in women $\left(P=3.6 \times 10^{-4}\right)$ but with decreased waist circumference in men $\left(P=6.8 \times 10^{-3}\right)$. These differences in the relationships between waist circumference, hip circumference and WHR underlie some of the sexual dimorphism in the patterns of WHR association.

\section{Enrichment of association with metabolic traits}

We evaluated the 14 WHR-associated loci for their relationships with related metabolic traits using GWAS data provided by trait-specific consortia ${ }^{19}{ }^{21}$ as well as our de novo genotyped follow-up studies. As expected given the sample overlap between this GWAS data and our WHR GWAS data as well as information on known trait correlations (Supplementary Table 4), we observed directionally consistent enrichment of associations ( $P$ $<0.05$ ) between the 14 WHR-associated alleles and increased triglycerides, low-density lipoprotein (LDL) cholesterol, fasting insulin and homeostasis model assessment (HOMA)derived measures of insulin resistance (binomial $P$ from $3.2 \times 10^{-4}$ to $1.8 \times 10^{-8}$; Table 3 and Supplementary Table 5). For example, the WHR-increasing allele at GRB14 showed strong associations with increased triglycerides $\left(P=7.4 \times 10^{-9}\right)$, fasting insulin levels $(P=$ $\left.5.0 \times 10^{-6}\right)$ and insulin resistance $\left(P=1.9 \times 10^{-6}\right)$. Eleven of the 14 WHR-associated loci showed directionally consistent associations with T2D, with three of these loci (at ADAMTS9, NISCH-STAB1 and ITPR2-SSPN) reaching nominal significance $(P<0.05)$ (Table 3 and Supplementary Table 5). Because the association signals for correlated traits in this analysis were vulnerable to overestimation given the overlap in the GWAS samples examined, we repeated these analyses and restricted the samples included to those from our de novo genotyped follow-up studies. Although this also resulted in a lower sample size, similar patterns of enrichment were still observed (Supplementary Table 5).

\section{Pathway analysis and potential biological roles}

To identify potential functional connections and pathway relationships between genes mapping at the WHR-associated loci, we focused on the 95 genes located in a 2-Mb interval centered around each of the 48 independent SNPs that attained $P<1.0 \times 10^{-5}$ in the WHR discovery studies.

First, we performed a survey of the published literature using GRAIL ${ }^{22}$ to search for connectivity between the genes and specific keywords that describe these functional connections (Online Methods). Although there was no evidence after correcting for multiple testing that the connectivity between these genes was greater than chance, we identified eight genes with nominal significance $(P<0.05)$ for potential functional connectivity (PLXND, HOXC10, TBX15, RSPO3, HOXC4, HOXC6, KREMEN1 and HOXC11). The keywords associated with these connections included 'vegf', 'homeobox', 'patterning', 'mesenchyme', 'embryonic', 'development' and 'angiogenesis'. 
Additionally, we performed pathway analyses using the PANTHER database ${ }^{23}$ based on the same set of 95 genes (Online Methods and Supplementary Note). This analysis generated some evidence for over-representation of 'developmental processes' $\left(P=5.8 \times 10^{-8}\right)$ and 'mRNA transcription regulation' $\left(P=2.7 \times 10^{-6}\right)$ but neither of these factors retained nominal significance after adjustment for bias (for example, due to non-random SNP coverage in relation to genes) and the number of biological processes tested (Supplementary Note and Supplementary Table 6).

Finally, we examined the described functional roles of some of the most compelling candidates based on either proximity to the signal or the other analyses described in this paper. These analyses uncovered possible genetic roles in adipocyte development (TBX15), pattern formation during embryonic development (HOXC13), angiogenesis ( VEGFA, RSPO3 and STAB1), Wnt and $\beta$-catenin signaling (RSPO3 and KREMEN1), insulin signaling ( $A D A M T S 9$, GRB14 and NISCH), lipase activity ( $L Y P L A L 1)$, lipid biosynthesis (PIGC) and intracellular calcium signaling (ITPR2) (Supplementary Note).

\section{Evaluation of copy number variants and non-synonymous changes}

Both common and rare copy number variants (CNVs) have been reported to be associated with overall adiposity ${ }^{14},{ }^{15},{ }^{24},{ }^{25}$, but the impact of $\mathrm{CNVs}$ on fat distribution has not been evaluated previously. To examine the potential contribution of common CNVs to variation in WHR, we looked for evidence of association in our genome-wide association discovery meta-analysis using a set of 6,018 CNV-tagging SNPs which collectively capture $>40 \%$ of common CNVs that are greater than $1 \mathrm{~kb}$ in length ${ }^{26},{ }^{27}$ (Online Methods and Supplementary Note).

One CNV-tagging SNP (rs1294421 in LY86) was observed among our 14 WHR-associated loci. This SNP is in strong $\mathrm{LD}\left(r^{2}=0.98\right)$ with a 2,832 -bp duplication variant $\left(\right.$ CNVR2760.1) ${ }^{27}$ located $12 \mathrm{~kb}$ from an expressed sequence tag (BC039678) and $87 \mathrm{~kb}$ from $L Y 86$ such that the duplication allele is associated with reduced WHR. The duplicated region consists entirely of noncoding sequence but includes part of a predicted enhancer sequence (E.5552.1) $)^{28}$.

To identify other putatively causal variants in our associated regions, we searched for nonsynonymous coding SNPs in strong LD (defined as $r^{2}>0.7$ ) with the most strongly associated SNPs at each locus using data from the HapMap (Build 21) and 1000 Genomes Project (April and August 2009 releases). In this search, one lead SNP (rs6784615, at the NISCH-STAB 1 locus) was correlated with non-synonymous changes in two nearby genes, DNAH1 (p.Val441Leu, p.Arg1285Trp and p.Arg3809Cys) and GLYCTK (p.Leu170Val). Fine-mapping and functional studies will be required to determine whether the $D N A H 1$ or GLYCTK SNPs or the $L Y 86 \mathrm{CNV}$ are causal for the WHR associations at these loci.

\section{Effect of WHR associations on expression in relevant tissues}

Expression quantitative trait locus (eQTL) data can implicate regional transcripts that mediate trait associations, and we therefore examined the 14 WHR-associated loci using eQTL data from human subcutaneous adipose tissue (SAT) ${ }^{29}$ (two separate sample sets, $n=$ 610 and $n=603)$, omental fat ${ }^{30}(n=740)$, $\operatorname{liver}^{30}(n=518)$, $\operatorname{blood}^{29}(n=745)$ and lymphocytes $^{31}(n=830)$ (Online Methods and Supplementary Note).

At six of the loci, the WHR-associated SNP was either the strongest SNP associated with significant $\left(P<1.0 \times 10^{-5}\right)$ expression of a local (within $1 \mathrm{Mb}$ ) gene transcript or explained the majority of the association between the most significant eQTL SNP and the gene transcript in conditional analyses (adjusted $P>0.05$; Table 4). For example, the WHRassociated SNP rs1011731 (near DNM3-PIGC) was strongly associated with expression of 
$P I G C$ in lymphocytes $\left(P=5.9 \times 10^{-10}\right)$; furthermore, rs 1011731 is in high $\operatorname{LD}\left(r^{2}=1.00, D^{\prime}\right.$ $=1.00$ from the HapMap CEU population) with the SNP with the strongest effect on PIGC expression (rs991790), and this cis eQTL association was abolished by conditioning on rs1011731. These analyses therefore indicate that these two signals are coincident and that $P I G C$ is a strong candidate for mediating the WHR association at rs 1011731 . We found similar evidence for coincidence of the WHR signal with expression for rs984222 (TBX15 in omental fat), rs 1055144 (expressed sequence tag AA553656 in SAT), rs10195252 (GRB14 in SAT), rs4823006 (ZNRF3 in SAT and omental fat) and rs6784615 (STAB1 in blood) (Table 4). Taken together, the overlap between trait association and gene expression at these loci suggests that the WHR associations may be driven through altered expression of PIGC, TBX15, AA553656, GRB1, ZNRF3 and STAB1.

\section{RNA expression of gluteal and abdominal fat tissue}

To determine whether genes within the WHR-associated loci showed evidence of differential transcription in distinct fat depots, we compared expression levels in gluteal or abdominal SAT in 49 individuals. We focused on the 15 genes with the strongest credentials for causal involvement (on the basis of proximity to the lead SNP and/or other biological or functional data; Table 1) for which expression data were available. Five of these genes (RSPO3, TBX15, ITPR2, WARS2 and STAB1) were differentially expressed between the two tissues (using an $F$ test, corrected for false discovery rate across the 15 expressed genes, $P<0.05$; Supplementary Table 7). This supports the hypothesis that, at some loci at least, the association with WHR reflects depot-specific differences in expression patterns.

\section{DISCUSSION}

Overall, our findings demonstrate that the genetic regulation of body fat distribution involves loci and processes that are largely distinct from those that influence BMI and risk of obesity. This finding is consistent with the evidence that WHR displays substantial heritability even after adjustment for BMI. The loci that emerged from this study display no overlap with those shown to be associated with BMI either in previous reports ${ }^{14}{ }_{-} 16$ or in the expanded meta-analysis recently completed by the GIANT consortium ${ }^{32}$.

Another point of distinction between our findings and those for BMI relates to the evidence for sexual dimorphism that we observed at several of the WHR-associated loci. Sex differences in the regulation of body fat distribution have long been acknowledged without a clear understanding of the underlying molecular mechanisms. These differences become apparent during puberty and are generally attributed to the influence of sex hormones ${ }^{33}$. Consistent with our findings, variance decomposition studies have shown that the genetic contribution to the overall variance in WHR, waist and hip circumference is greater in women ${ }^{17}$. Although there is some evidence for loci with differential sex effects influencing lipids $^{34}$, uric acid levels ${ }^{35}$ and risk of schizophrenia ${ }^{36}$, we are unaware of prior reports indicating such strong enrichment of female-specific associations for any other phenotype, including $\mathrm{BMI}^{32}$.

The primary objective of genetic discovery efforts is to characterize the specific mechanisms involved in regulating the trait of interest. Despite the considerable challenges associated with moving from common variant association signals to defining causal alleles and pathways, we have identified strong candidates at several of the loci. For example, the cis eQTL data implicate GRB14 as a compelling candidate for the WHR association on chromosome 2, and we were able to show that the same GRB14 variants are also associated with triglyceride and insulin levels, consistent with previous association of this locus with high-density lipoprotein (HDL) cholesterol ${ }^{37}$. These inferences about the role of GRB14 are supported by evidence that Grb14-deficient mice exhibit improved glucose homeostasis 
despite lower circulating insulin levels, as well as enhanced insulin signaling in liver and

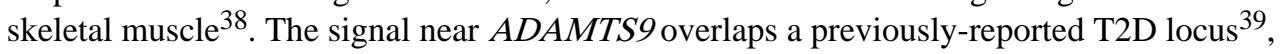
and the lead SNP for WHR in our study is identical to the SNP displaying the strongest T2D association in a previous expanded T2D meta-analysis ${ }^{40}$. Given evidence that $A D A M T S 9$ T2D risk alleles are associated with insulin resistance in peripheral tissues ${ }^{41}$, these findings are consistent with a primary effect of $A D A M T S 9$ variants on body fat distribution. At the chromosome 6 locus, VEGFA is the most apparent biological candidate given the presumed role of $V E G F A$ as a mediator of adipogenesis ${ }^{42}$ and evidence that serum levels of VEGFA are correlated with obesity ${ }^{43},{ }^{44}$. Finally, at the TBX15-WARS 2 locus, TBX15 emerges as the strongest candidate based on the cis eQTL data in omental fat, marked depot-specific differences in adipose tissue expression in mice and humans and associations between $T B X 15$ expression in visceral fat and $\mathrm{WHR}^{45},{ }^{46}$.

Our efforts to use pathway- and literature-mining approaches to look for functional enrichment of the genes mapping to associated regions met with only limited success but did provide some support for over-representation of developmental processes. Developmental genes have been implicated in fat accumulation and distribution ${ }^{45},{ }^{46}$, and recent evidence supports a link between developmental genes, including $\mathrm{HOXC13}$ (ref. ${ }^{47}$ ) and $T B X 15$ (refs. $45,{ }^{48}$ ), and body fat distribution. Developmental genes may in part determine the adipocytespecific expression patterns that have been observed in different fat depots ${ }^{45}$. Taken together, our findings point to a set of genes influencing body fat distribution that have their principal effects in adipose tissue. This is in contrast to the predominantly central (hypothalamic) processes that are involved in the regulation of BMI and overall adiposity 49 .

By providing new insights into the regulation of body fat distribution, the present study raises a number of issues for future investigation. From the genetic perspective, resequencing, dense-array genotyping and fine-mapping approaches will be required to characterize causal variants at the loci we have identified and to support further discoveries that may account for the substantial proportion of genetic variance unexplained by our findings. From the clinical perspective, it will be important to explore the relationship of these variants to more refined measures of body fat distribution derived from detailed imaging studies, to use the variants identified to characterize the causal relationships between body fat distribution and related metabolic and cardiovascular traits and to explore population differences in patterns of body fat distribution. Efforts to tackle overall obesity through therapeutic or lifestyle-based modulation of overall energy balance have proved extremely challenging to implement, and the manipulation of processes associated with more beneficial patterns of fat distribution offers an alternative perspective for future drug discovery.

\section{METHODS}

Methods and any associated references are available in the online version of the paper at http://www.nature.com/naturegenetics/.

\section{Supplementary Material}

Refer to Web version on PubMed Central for supplementary material.

\section{Footnotes}

Correspondence should be addressed to C.M.L. (celi@well.ox.ac.uk), K.L.M. (mohlke@med.unc.edu), C.S.F. (foxca@nhlbi.nih.gov), M.I.M. (mark.mccarthy@drl.ox.ac.uk) or I.M.H. (iris.heid@klinik.uni-regensburg.de). 
${ }^{214}$ These authors contributed equally to this work.

${ }^{215}$ These authors jointly directed this work.

AUTHOR CONTRIBUTIONS Writing group: I.B., C.S.F., I.M.H. (lead), C.M.L. (lead), M.I.M., K.L. Mohlke, L.Q., V. Steinthorsdottir, G.T., M.C.Z.

Waist phenotype working group: T.L.A., N.B., I.B., L.A.C., C.M.D., C.S.F., T.B.H., I.M.H., A.U.J., C.M.L. (lead), R.J.F.L., R.M., M.I.M., K.L. Mohlke, L.Q., J.C.R., E.K.S., V. Steinthorsdottir, K. Stefansson, G.T., U.T., C.C.W., T.W., T.W.W., H.E.W., M.C.Z.

Data cleaning and analysis: S.I.B., I.M.H. (lead), E.I., A.U.J., H.L., C.M.L. (lead), R.J.F.L. (lead), J.L., R.M., L.Q., J.C.R., E.K.S., G.T., S.V., M.N.W., E.W., C.J.W., T.W.W., T.W.

Sex-specific analyses: S.I.B., T.E., I.M.H., A.U.J., T.O.K., Z.K., S.L., C.M.L., R.J.F.L., R.M., K.L. Monda, K.E.N., L.Q., J.C.R. (lead), V. Steinthorsdottir, G.T., T.W.W. (lead).

eQTL and expression analyses: S.I.B., A.L.D., C.C.H., J.N.H., F.K., L.M.K., C.M.L., L.L., R.J.F.L., J.L., M.F.M., J.L.M., C.M., G.N., E.E.S., E.K.S., V. Steinthorsdottir, G.T., K.T.Z.

Pathway and CNV analyses: C.M.L., S.A.M., M.I.M., J.N., V. Steinthorsdottir, G.T., B.F.V

Secondary analyses: S.I.B., I.B.B., N.C., K.E., T.M.F., M.F.F., T.F., M.E.G., J.N.H., E.I., G.L., C.M.L., H.L., R.M., M. Mangino, M.I.M., K.L. Mohlke, D.R.N., J.R.O., S.P., J.R.B.P., J.C.R., A.V.S., E.K.S., P.M.V., M.N.W., C.J.W., R.J.W., E.W., A.R.W., J.Y

Study-specific analyses: G.R.A., D.A., N.A., T.A., T.L.A., N.B., C.C., P.S.C., L.C., L.A.C., D.I.C., M.N.C., C.M.D., T.E., K.E., E.F., M.F.F., T.F., A.P.G., N.L.G., M.E.G., C. Hayward, N.L.H., I.M.H., J.J.H., A.U.J., Å.J., T. Johnson, J.O.J., J.R.K., M. Kaakinen, K. Kapur, S. Ketkar, J.W.K., P. Kraft, A.T.K., Z.K., J. Kettunen, C. Lamina, R.J.F.L., C. Lecoeur, H.L., M.F.L., C.M.L., J.L., R.W.L., R.M., M. Mangino, B.M., K.L. Monda, A.P.M., N.N., K.E.N., D.R.N., J.R.O., K.K.O., C.O., M.J.P., O. Polasek, I. Prokopenko, N.P., M.P., L.Q., J.C.R., N.W.R., S.R., F.R., N.R.R., C.S., L.J.S., K. Silander, E.K.S., K. Stark, S.S., A.V.S., N.S., U.S., V. Steinthorsdottir, D.P.S., I.S., M.L.T., T.M.T., N.J.T., A.T., G.T., A.U., S.V., V. Vitart, L.V., P.M.V., R.M.W., R.W., R.J.W., S.W., M.N.W., C.C.W., C.J.W., T.W.W., A.R.W., J.Y., J.H.Z., M.C.Z.

Study-specific genotyping: D.A., T.L.A., L.D.A., N.B., I.B., A.J.B., E.B., L.L.B., I.B.B., H.C., D.I.C., I.N.M.D., M. Dei, M.R.E., P.E., K.E., N.B.F., M.F., A.P.G., H.G., C.G., E.J.C.G., C.J.G., T. Hansen, A.L.H., N.H., C. Hayward, A.A.H., J.J.H., F.B.H., D.J.H., J.H., W.I., M.R.J., Å.J., J.O.J., J.W.K., P. Kovacs, A.T.K., H.K.K., J. Kettunen, P. Kraft, R.N.L., C.M.L., R.J.F.L., J.L., M.L.L., M.A.M., M. Mangino, W.L.M., M.I.M., J.B.J.M., M.J.N., M.N., D.R.N., K.K.O., C.O., O. Pedersen, L.P., M.J.P., G.P., A.N.P., N.P., L.Q., N.W.R., F.R., N.R.R., C.S., A.J.S., N.S., A.C.S., M.T., B.T., A.U., G.U., V. Vatin, P.M.V., H.W., P.Z.

Study-specific phenotyping: H.A., P.A., D.A., A.M.A., T.L.A., B.B., S.R.B., R.B., E.B., I.B.B., J.P.B., M. Dörr, C.M.D., P.E., M.F.F., C.S.F., T.M.F., M.F., S.G., J.G., L.C.G., T. Hansen, A.S.H., C. Hengstenberg, A.L.H., A.T.H., K.H.H., A. Hofman, F.B.H., D.J.H., B.I., T.I., T. Jørgensen, P.J., M.R.J., Å.J., A.J., A.L.J., J.O.J., F.K., L.K., J. Kuusisto, K. Kvaloy, R.K., S. Ketkar, J.W.K., I.K., S. Koskinen, V.K., M. Kähönen, P. Kovacs, O.L., R.N.L., B.L., J.L., G.M.L., R.J.F.L., T.L., M. Mangino, M.I.M., C.O., B.M.P., O. Pedersen, C.G.P.P., J.F.P., I. Pichler, K.P., O. Polasek, A.P., L.Q., M.R., I.R., O.R., V. Salomaa, J. Saramies, P.E.H.S., K. Silander, N.J.S., J.H.S., T.D.S., D.P.S., R.S., H.M.S., J. Sinisalo, T.T., A.T., M.U., P.V., C.B.V., L.V., J.V., D.R.W., G.B.W., S.H.W., G.W., J.C.W., A.F.W., L.Z., P.Z.

Study-specific management: G.R.A., A.M.A., B.B., Y.B.S., R.N.B., H.B., J.S.B., S.B., M.B., E.B., D.I.B., I.B.B., J.P.B., M.J.C., F.S.C., L.A.C., G.D., C.M.D., S.E., G.E., P.F., C.S.F., T.M.F., L.C.G., V.G., U.G., M.E.G., T. Hansen, C. Hengstenberg, K.H., A. Hamsten, T.B.H., A.T.H., A. Hofman, F.B.H., D.J.H., B.I., T.I., C.I., T. Jørgensen, M.R.J., A.L.J., F.K., K.T.K., W.H.L.K., R.K., J. Kaprio, M. Kähönen, M.L., D.A.L., L.J.L., C.M.L., R.J.F.L., T.L., M. Marre, T.M., A.M.E.T., K.M., M.I.M., K.L. Mohlke, P.B.M., K.E.N., M.S.N., D.R.N., B.O., C.O., O. Pedersen, L.P., B.W.P., P.P.P., B.M.P., L.J.P., T.Q., A.R., I.R., O.R., P.M.R., V. Salomaa, P.S., D.S., A.R.S., N.S., T.D.S., K. Stefansson, D.P.S., A.C.S., M.S., T.T., J.T., U.T., A.T., M.U., A.U., T.T.V., P.V., H.V., J.V., P.M.V., N.J.W., H.E.W., J.F.W., J.C.W., A.F.W

Steering committee: G.R.A., T.L.A., I.B., S.I.B., M.B., I.B.B., P.D., C.M.D., C.S.F., T.M.F., L.C.G., T. Haritunians, J.N.H. (chair), D.J.H., E.I., R.K., R.J.F.L., M.I.M., K.L. Mohlke, K.E.N., J.R.O., L.P., D.S., D.P.S., U.T., H.E.W.

Note: Supplementary information is available on the Nature Genetics website.

URLs. LocusZoom, http://csg.sph.umich.edu/locuszoom/.

COMPETING FINANCIAL INTERESTS The authors declare competing financial interests: details accompany the full-text HTML version of the paper at http://www.nature.com/naturegenetics/.

Reprints and permissions information is available online at http://npg.nature.com/reprintsandpermissions/.

\section{Acknowledgments}

Funding for this study was provided by the Academy of Finland (grants 104781, 120315, 129269, 117797, 121584, 126925, 129418, 129568, 77299, 124243, 213506, 129680, 129494, 10404, 213506, 129680, 114382, 126775. 127437, 129255, 129306, 130326, 209072, 210595, 213225 and 216374); an ADA Mentor-Based Postdoctoral Fellowship grant; Affymetrix, Inc., for genotyping services (N02-HL-6-4278); ALF/LUA Gothenburg; Althingi (the Icelandic Parliament); Amgen; AstraZeneca AB; Augustinus Foundation; Becket Foundation; Biocentrum 
Helsinki; Biomedicum Helsinki Foundation; Boston Obesity Nutrition Research Center (DK46200); British Diabetes Association (1192); British Diabetic Association Research; British Heart Foundation (97020, PG/02/128); Busselton Population Medical Research Foundation; Cambridge NIHR Comprehensive Biomedical Research Centre; CamStrad; Chief Scientist Office of the Scottish Government; Contrat Plan Etat Région de France; Danish Centre for Health Technology Assessment; Danish Diabetes Association; Danish Ministry of Internal Affairs and Health; Danish Heart Foundation; Danish Pharmaceutical Association; Danish Research Council; DIAB Core (German Network of Diabetes); Diabetes UK; Donald W. Reynolds Foundation; Dresden University of Technology Funding Grant, Med Drive; EMGO+ institute; Emil and Vera Cornell Foundation; Erasmus Medical Center and Erasmus University, Rotterdam, The Netherlands; Estonian Government SF0180142s08; European Commission (2004310, 212111, 205419, 245536, DG XII, HEALTH-F4-2007-201413, FP7/2007-2013, QLG1-CT-2000-01643, QLG2-CT-2002-01254, LSHG-CT-2006-018947, LSHG-CT-2006-01947, LSHG-CT-2004-512066, LSHMCT-2007-037273, EU/WLRT-2001-01254, LSHG-CT-2004-518153, SOC 95201408 05F02, Marie Curie IntraEuropean Fellowship); Federal Ministry of Education and Research, Germany (01ZZ9603, 01ZZ0103, 01ZZ0403, 03ZIK012, 01 EA 9401); Federal State of Mecklenburg-West Pomerania; Finnish Diabetes Research Foundation; Finnish Diabetes Research Society; Finnish Foundation for Pediatric Research; Finnish Foundation of Cardiovascular Research; Finnish Medical Society; Finska Läkaresällskapet; Finnish Ministry of Education; Folkhälsan Research Foundation; Fond Européen pour le Développement Régional; Fondation LeDucq; Foundation for Life and Health in Finland; GEN-AU 'GOLD' from Austria; German Bundesministerium fuer Forschung und Technology (\# 01 AK 803 A-H, \# 01 IG 07015 G); German National Genome Research Net NGFN2 and NGFNplus (01GS0823, FKZ 01GS0823); German Research Council (KFO-152); GlaxoSmithKline; Göteborg Medical Society; Gyllenberg Foundation; Health Care Centers in Vasa, Närpes and Korsholm; Healthway, Western Australia; Helmholtz Center Munich; Helsinki University Central Hospital; Hjartavernd (the Icelandic Heart Association); Ib Henriksen Foundation; IZKF (B27); Jalmari and Rauha Ahokas Foundation; Juho Vainio Foundation; Juvenile Diabetes Research Foundation International (JDRF); Karolinska Institute and the Stockholm County Council (560183); Knut and Alice Wallenberg Foundation; Lundbeck Foundation Centre of Applied Medical Genomics for Personalized Disease Prediction, Prevention and Care; Knut Krohn, Microarray Core Facility of the Interdisciplinary Centre for Clinical Research (IZKF), University of Leipzig, Germany; Lundberg Foundation; MC Health; Ministry of Cultural Affairs of the Federal State of Mecklenburg-West Pomerania, Germany; South Tyrol Ministry of Health; Ministry of Science, Education and Sport of the Republic of Croatia (216-1080315-0302); Medical Research Council UK (G0000649, G0601261, G9521010D, G0000934, G0500539, G0600331, PrevMetSyn); Montreal Heart Institute Foundation; MRC Centre for Obesity-Related Metabolic Disease; Municipal Health Care Center and Hospital in Jakobstad; Municipality of Rotterdam; Närpes Health Care Foundation; National Health and Medical Research Council of Australia and the Great Wine Estates Auctions; Netherlands Centre for Medical Systems Biology (SPI 56-464-1419); Netherlands Ministry for Health, Welfare and Sports; Netherlands Ministry of Education, Culture and Science; Netherlands Genomics Initiative; Netherlands Consortium for Healthy Aging (050-060-810); Netherlands Organisation of Scientific Research Netherlandse Organisatie voor Wetenschappelijk Onderzoek (NWO) Investments (175.010.2005.011, 911-03-012, 904-61-090, 904-61-193, 480-04-004, 400-05-717); National Institute on Aging Intramural Research Program; US National Institutes of Health (CA047988, CA65725, CA87969, CA49449, CA67262, CA50385, DK075787, DK062370, DK58845, DK072193, K23-DK080145, K99HL094535, N01-HC85079 through N01-HC85086, N01-HG-65403, N01-AG-12100, N01-HC-25195, N01-HC35129, N01-HC15103, N01-HC55222, N01-HC75150, N01-HC45133, N01-HC55015, N01-HC55016, N01-HC-55018, N01-HC-55019, N01-HC-55020, N01-HC-55021, N01HC-55022, NO1-AG-1-2109, HL71981, HG005581, HG002651, HL084729, HL043851, HHSN268200625226C, K23-DK080145, MH084698, P30-DK072488, R01-DK075787, R01 HL087652, R01-HL087641, R01-HL59367, R01-HL086694, R01-HL087647, R01-HL087679, R01-HL087700, R01-AG031890, R01-HL088119, R01DK068336, R01-DK075681, R01-DK-073490, R01-DK075787, R01-MH63706, U01-HL72515, U01-GM074518, U01-HL084756, U01-HG004399, UO1-CA098233, UL1-RR025005, UL1-RR025005, U01-HG004402, U01DK062418, U01 HL080295, T32-HG00040, 263-MA-410953, 1RL1-MH083268-01, intramural project 1Z01HG000024); National Institute for Health Research (NIHR); Neuroscience Campus Amsterdam; Novo Nordisk Foundation; Novo Nordisk Inc., Research Foundation of Copenhagen County; Ollqvist Foundation; Paavo Nurmi Foundation; Päivikki and Sakari Sohlberg Foundation; Pew Scholarship for the Biomedical Sciences; Perklén Foundation; Petrus and Augusta Hedlunds Foundation; Research Institute for Diseases in the Elderly (014-93-015, RIDE, RIDE2); Sahlgrenska Center for Cardiovascular and Metabolic Research (CMR, A305:188); Siemens Healthcare, Erlangen, Germany; Signe and Ane Gyllenberg Foundation; Sigrid Juselius Foundation; Social Insurance Institution of Finland; Social Ministry of the Federal State of Mecklenburg-West Pomerania, Germany; South Tyrolean Sparkasse Foundation; State of Bavaria, Germany; Support for Science Funding programme; Swedish Cultural Foundation in Finland; Swedish Foundation for Strategic Research (SSF); Swedish Heart-Lung Foundation; Swedish Medical Research Council (8691, K2007-66X-20270-01-3, K2010-54X-09894-19-3); Swedish Society of Medicine; Swiss National Science Foundation (33CSCO-122661); the Royal Society; the Royal Swedish Academy of Science; Torsten and Ragnar Söderberg's Foundation; Turku University Hospitals; UK Department of Health Policy Research Programme; University and Research of the Autonomous Province of Bolzano; University Hospital Medical funds to Tampere; University Hospital Oulu, Biocenter, University of Oulu, Finland (75617); Västra Götaland Foundation; Wellcome Trust (077016/Z/05/Z, 068545/Z/02, 072960, 076113, 083270, 085301, 079557, 081682, 075491, 076113/B/04/Z, 091746/Z/10/Z, 079895, WT086596/Z/08/Z, WT Research Career Development Fellowship; WT Career Development Award); Western Australian Genetic 
Epidemiology Resource and the Western Australian DNA Bank (both National Health and Medical Research Council of Australia Enabling Facilities); Yrjö Jahnsson Foundation.

\section{References}

1. Carey VJ, et al. Body fat distribution and risk of non-insulin-dependent diabetes mellitus in women. The Nurses' Health Study. Am. J. Epidemiol. 1997; 145:614-619. [PubMed: 9098178]

2. Wang Y, Rimm EB, Stampfer MJ, Willett WC, Hu FB. Comparison of abdominal adiposity and overall obesity in predicting risk of type 2 diabetes among men. Am. J. Clin. Nutr. 2005; 81:555563. [PubMed: 15755822]

3. Canoy D. Distribution of body fat and risk of coronary heart disease in men and women. Curr. Opin. Cardiol. 2008; 23:591-598. [PubMed: 18830075]

4. Pischon T, et al. General and abdominal adiposity and risk of death in Europe. N. Engl. J. Med. 2008; 359:2105-2120. [PubMed: 19005195]

5. Snijder MB, et al. Associations of hip and thigh circumferences independent of waist circumference with the incidence of type 2 diabetes: the Hoorn Study. Am. J. Clin. Nutr. 2003; 77:1192-1197. [PubMed: 12716671]

6. Snijder MB, et al. Trunk fat and leg fat have independent and opposite associations with fasting and postload glucose levels: the Hoorn study. Diabetes Care. 2004; 27:372-377. [PubMed: 14747216]

7. Mills GW, et al. Heritability estimates for beta cell function and features of the insulin resistance syndrome in UK families with an increased susceptibility to type 2 diabetes. Diabetologia. 2004; 47:732-738. [PubMed: 15298351]

8. Souren NY, et al. Anthropometry, carbohydrate and lipid metabolism in the East Flanders Prospective Twin Survey: heritabilities. Diabetologia. 2007; 50:2107-2116. [PubMed: 17694296]

9. Rose KM, Newman B, Mayer-Davis EJ, Selby JV. Genetic and behavioral determinants of waist-hip ratio and waist circumference in women twins. Obes. Res. 1998; 6:383-392. [PubMed: 9845227]

10. Selby JV, et al. Genetic and behavioral influences on body fat distribution. Int. J. Obes. 1990; 14:593-602. [PubMed: 2228394]

11. Agarwal AK, Garg A. Genetic disorders of adipose tissue development, differentiation, and death. Annu. Rev. Genomics Hum. Genet. 2006; 7:175-199. [PubMed: 16722806]

12. Garg A. Acquired and inherited lipodystrophies. N. Engl. J. Med. 2004; 350:1220-1234. [PubMed: 15028826]

13. Lindgren CM, et al. Genome-wide association scan meta-analysis identifies three loci influencing adiposity and fat distribution. PLoS Genet. 2009; 5:e1000508. [PubMed: 19557161]

14. Thorleifsson G, et al. Genome-wide association yields new sequence variants at seven loci that associate with measures of obesity. Nat. Genet. 2009; 41:18-24. [PubMed: 19079260]

15. Willer CJ, et al. Six new loci associated with body mass index highlight a neuronal influence on body weight regulation. Nat. Genet. 2009; 41:25-34. [PubMed: 19079261]

16. Loos RJ, et al. Common variants near MC4R are associated with fat mass, weight and risk of obesity. Nat. Genet. 2008; 40:768-775. [PubMed: 18454148]

17. Zillikens MC, et al. Sex-specific genetic effects influence variation in body composition. Diabetologia. 2008; 51:2233-2241. [PubMed: 18839131]

18. Frayling TM, et al. A common variant in the FTO gene is associated with body mass index and predisposes to childhood and adult obesity. Science. 2007; 316:889-894. [PubMed: 17434869]

19. Kathiresan S, et al. Common variants at 30 loci contribute to polygenic dyslipidemia. Nat. Genet. 2009; 41:56-65. [PubMed: 19060906]

20. Dupuis J, et al. New genetic loci implicated in fasting glucose homeostasis and their impact on type 2 diabetes risk. Nat. Genet. 2010; 42:105-116. [PubMed: 20081858]

21. Saxena R, et al. Genetic variation in GIPR influences the glucose and insulin responses to an oral glucose challenge. Nat. Genet. 2010; 42:142-148. [PubMed: 20081857]

22. Raychaudhuri S, et al. Identifying relationships among genomic disease regions: predicting genes at pathogenic SNP associations and rare deletions. PLoS Genet. 2009; 5:e1000534. [PubMed: 19557189] 
23. Thomas PD, et al. PANTHER: a library of protein families and subfamilies indexed by function. Genome Res. 2003; 13:2129-2141. [PubMed: 12952881]

24. Bochukova EG, et al. Large, rare chromosomal deletions associated with severe early-onset obesity. Nature. 2010; 463:666-670. [PubMed: 19966786]

25. Walters RG, et al. A new highly penetrant form of obesity due to deletions on chromosome 16p11.2. Nature. 2010; 463:671-675. [PubMed: 20130649]

26. Conrad DF, et al. Origins and functional impact of copy number variation in the human genome. Nature. 2010; 464:704-712. [PubMed: 19812545]

27. Wellcome Trust Case Control Consortium. et al. Genome-wide association study of CNVs in 16,000 cases of eight common diseases and 3,000 shared controls. Nature. 2010; 464:713-720. [PubMed: 20360734]

28. Pennacchio LA, Loots GG, Nobrega MA, Ovcharenko I. Predicting tissue-specific enhancers in the human genome. Genome Res. 2007; 17:201-211. [PubMed: 17210927]

29. Emilsson V, et al. Genetics of gene expression and its effect on disease. Nature. 2008; 452:423428. [PubMed: 18344981]

30. Schadt EE, et al. Mapping the genetic architecture of gene expression in human liver. PLoS Biol. 2008; 6:e107. [PubMed: 18462017]

31. Dixon AL, et al. A genome-wide association study of global gene expression. Nat. Genet. 2007; 39:1202-1207. [PubMed: 17873877]

32. Speliotes EK, et al. Association analyses of 249,796 individuals reveal 18 new loci associated with body mass index. Nat. Genet. Oct 10.2010 advance online publication, doi:10.1038/ng.686. [PubMed: 20935630]

33. Wells JC. Sexual dimorphism of body composition. Best Pract. Res. Clin. Endocrinol. Metab. 2007; 21:415-430. [PubMed: 17875489]

34. Aulchenko YS, et al. Loci influencing lipid levels and coronary heart disease risk in 16 European population cohorts. Nat. Genet. 2009; 41:47-55. [PubMed: 19060911]

35. Döring A, et al. SLC $2 A 9$ influences uric acid concentrations with pronounced sex-specific effects. Nat. Genet. 2008; 40:430-436. [PubMed: 18327256]

36. Shifman S, et al. Genome-wide association identifies a common variant in the reelin gene that increases the risk of schizophrenia only in women. PLoS Genet. 2008; 4:e28. [PubMed: 18282107]

37. Ridker PM, et al. Polymorphism in the CETP gene region, HDL cholesterol, and risk of future myocardial infarction: genomewide analysis among 18,245 initially healthy women from the Women's Genome Health Study. Circ. Cardiovasc. Genet. 2009; 2:26-33. [PubMed: 20031564]

38. Cooney GJ, et al. Improved glucose homeostasis and enhanced insulin signalling in Grb14deficient mice. EMBO J. 2004; 23:582-593. [PubMed: 14749734]

39. Zeggini E, et al. Meta-analysis of genome-wide association data and large-scale replication identifies additional susceptibility loci for type 2 diabetes. Nat. Genet. 2008; 40:638-645. [PubMed: 18372903]

40. Voight BF, et al. Twelve type 2 diabetes susceptibility loci identified through large-scale association analysis. Nat. Genet. 2010; 42:579-589. [PubMed: 20581827]

41. Boesgaard TW, et al. Variant near ADAMTS9 known to associate with type 2 diabetes is related to insulin resistance in offspring of type 2 diabetes patients-EUGENE2 study. PLoS ONE. 2009; 4:e7236. [PubMed: 19789630]

42. Nishimura $S$, et al. Adipogenesis in obesity requires close interplay between differentiating adipocytes, stromal cells, and blood vessels. Diabetes. 2007; 56:1517-1526. [PubMed: 17389330]

43. Silha JV, Krsek M, Sucharda P, Murphy LJ. Angiogenic factors are elevated in overweight and obese individuals. Int. J. Obes. (Lond). 2005; 29:1308-1314. [PubMed: 15953938]

44. García de la Torre N, et al. Effects of weight loss after bariatric surgery for morbid obesity on vascular endothelial growth factor-A, adipocytokines, and insulin. J. Clin. Endocrinol. Metab. 2008; 93:4276-4281. [PubMed: 18713823]

45. Gesta S, et al. Evidence for a role of developmental genes in the origin of obesity and body fat distribution. Proc. Natl. Acad. Sci. USA. 2006; 103:6676-6681. [PubMed: 16617105] 
46. Gesta S, Tseng YH, Kahn CR. Developmental origin of fat: tracking obesity to its source. Cell. 2007; 131:242-256. [PubMed: 17956727]

47. Lanctôt C, Kaspar C, Cremer T. Positioning of the mouse Hox gene clusters in the nuclei of developing embryos and differentiating embryoid bodies. Exp. Cell Res. 2007; 313:1449-1459. [PubMed: 17346703]

48. Candille SI, et al. Dorsoventral patterning of the mouse coat by Tbx15. PLoS Biol. 2004; 2:E3. [PubMed: 14737183]

49. O'Rahilly S. Human genetics illuminates the paths to metabolic disease. Nature. 2009; 462:307314. [PubMed: 19924209] 


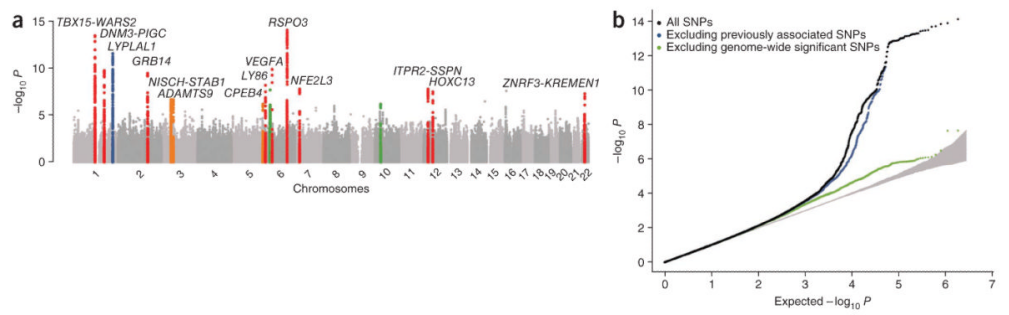

Figure 1.

Genome-wide association analyses for WHR in discovery studies. (a) Manhattan plot shows results of the WHR association meta-analysis in discovery studies (with $P$ values on the $y$ axis and the SNP genomic position on the $x$ axis). Colored genomic loci indicate significant association $\left(P<5 \times 10^{-8}\right)$ detected previously (blue) ${ }^{13}$, in our GWAS stage (red) and after the meta-analysis combining GWAS data with that from the follow-up studies (orange).

Two loci tested in the follow-up stage did not achieve genome-wide significance (green). (b) Quantile-quantile plot of SNPs for the discovery meta-analysis of WHR (black) and after removing SNPs within $1 \mathrm{Mb}$ of either the recently reported LYPLAL1 signal (blue) or the 14 significant associations (green). The gray area represents the $95 \% \mathrm{CI}$ around the test statistic under the null distribution. 

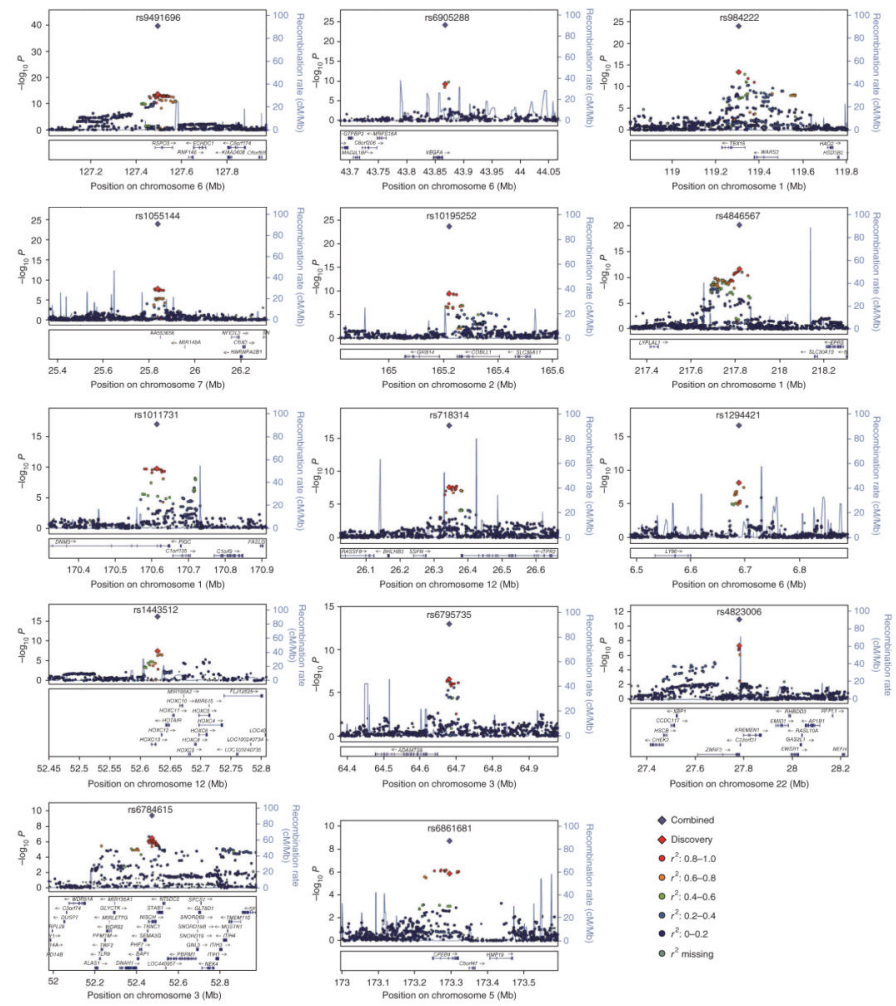

Figure 2.

Regional plots of 14 loci with genome-wide significant association. Shown is the SNP association with WHR in the meta-analysis of discovery studies for 14 loci (with $-\log _{10} P$ values on the $y$ axis and the SNP genomic position on the $x$ axis). In each panel, an index SNP is denoted with a purple diamond and plotted using the $P$ attained across discovery and follow-up data (Table 1). Estimated recombination rates are plotted in blue. SNPs are colored to reflect LD with the index SNP (pairwise $r^{2}$ values from HapMap CEU). Gene and microRNA annotations are from the UCSC genome browser. 

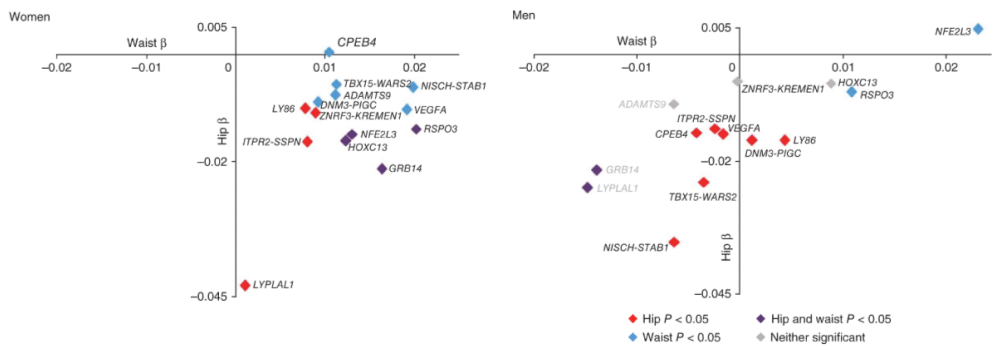

Figure 3.

Association of the 14 WHR loci with waist and hip circumference. $\beta$ coefficients for waist circumference (WC, $x$ axis) and hip circumference (HIP, $y$ axis) in women and men derived from the joint discovery and follow-up analysis. $P$ for WC and HIP are represented by color. In men, gray gene labels refer to those SNPs that were not significant in the male-specific WHR analysis. More details can be found in Supplementary Table 3. 


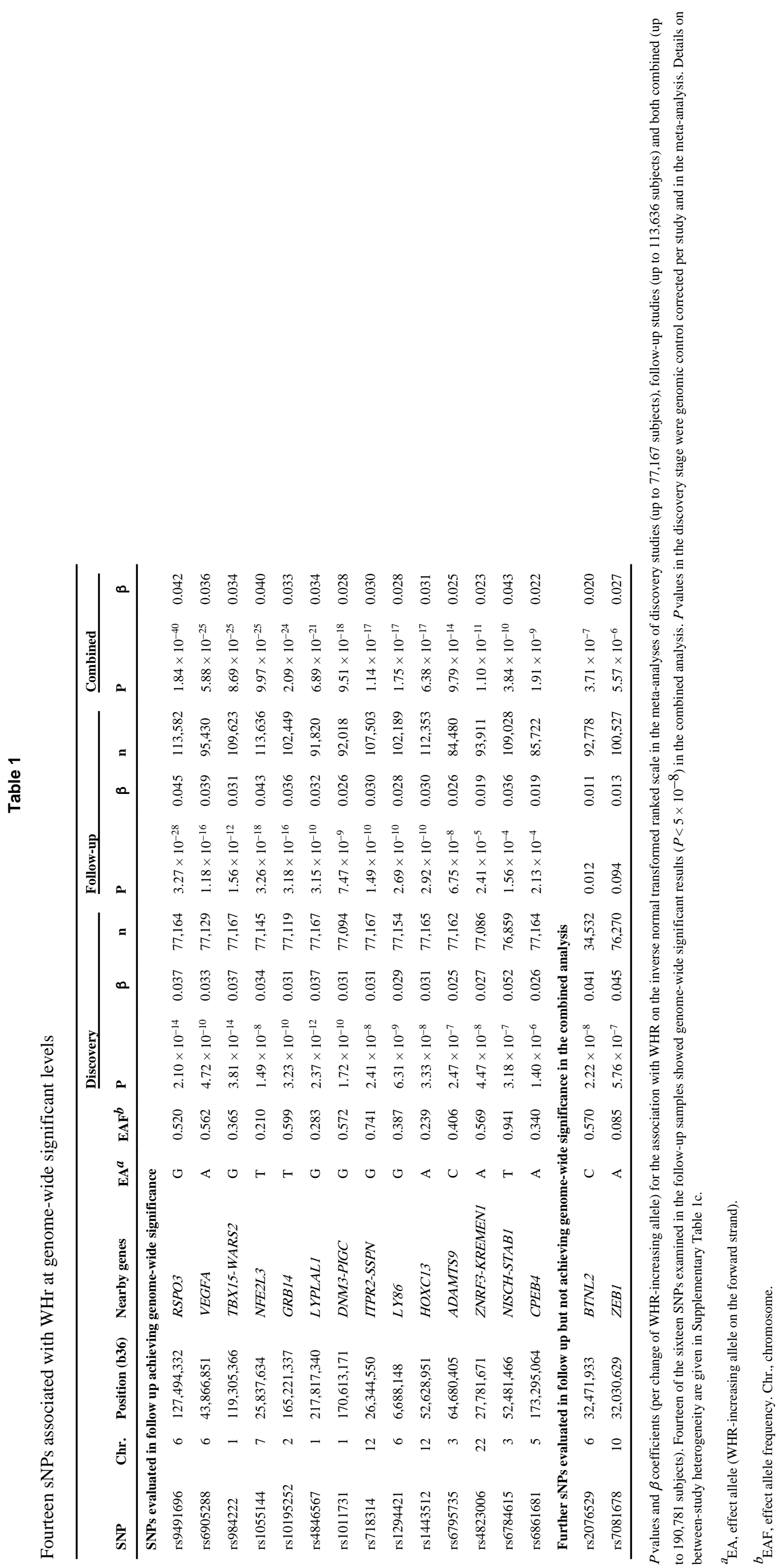




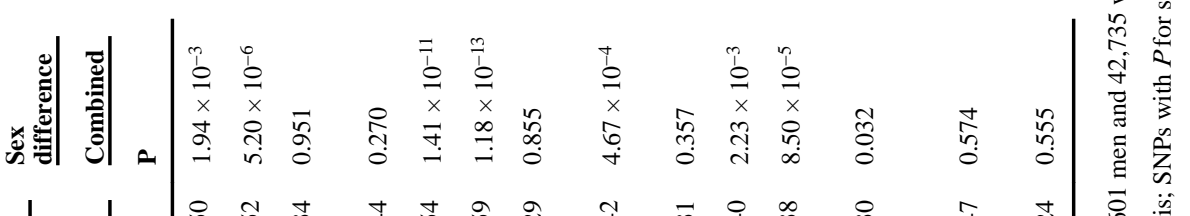

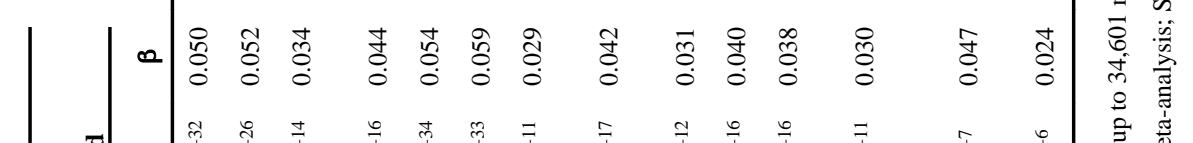

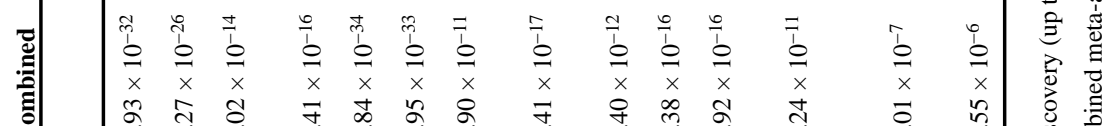

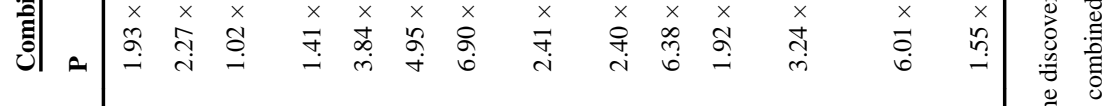

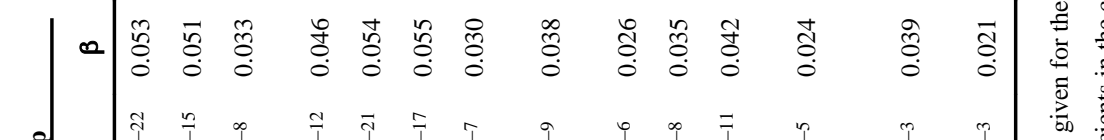

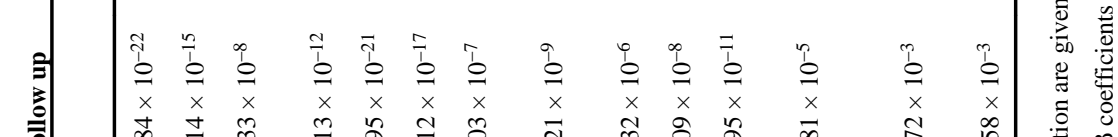

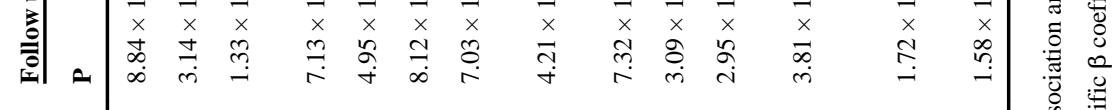

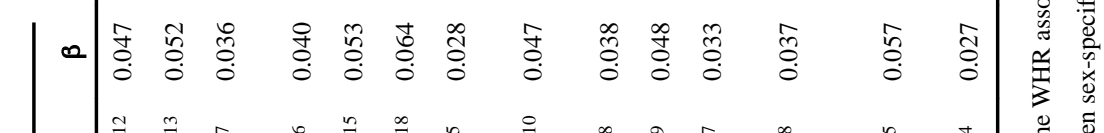

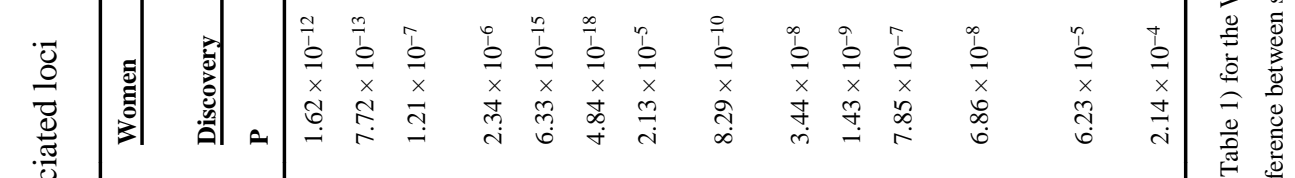
a

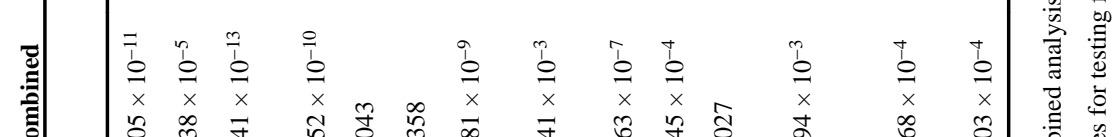

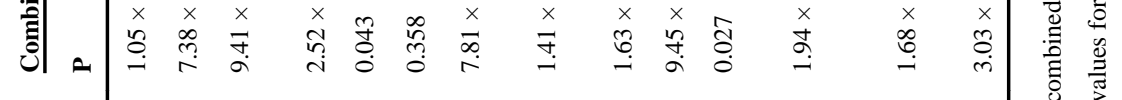

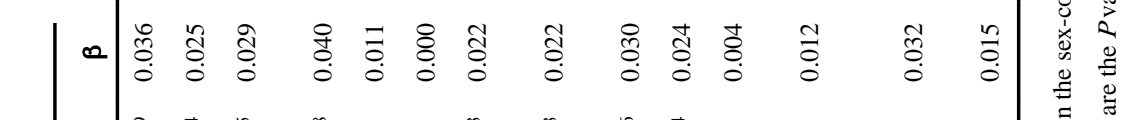

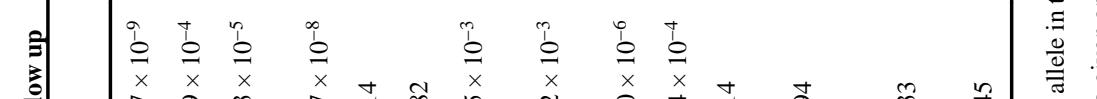

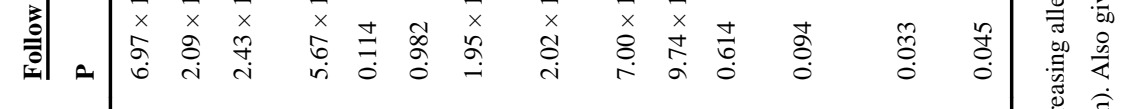

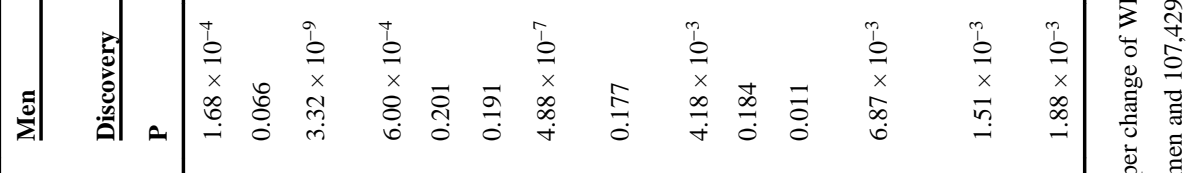

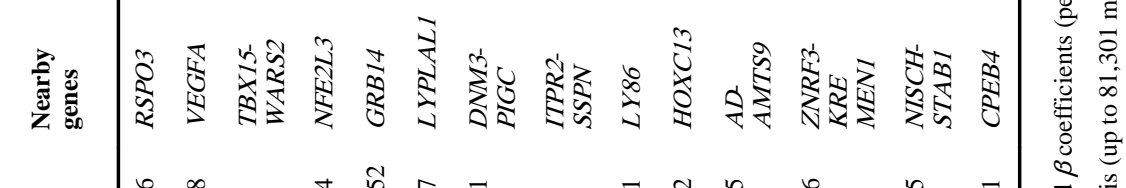

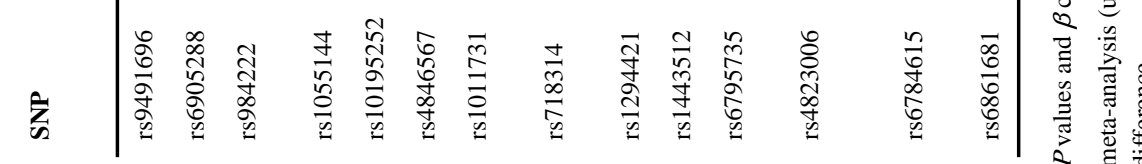




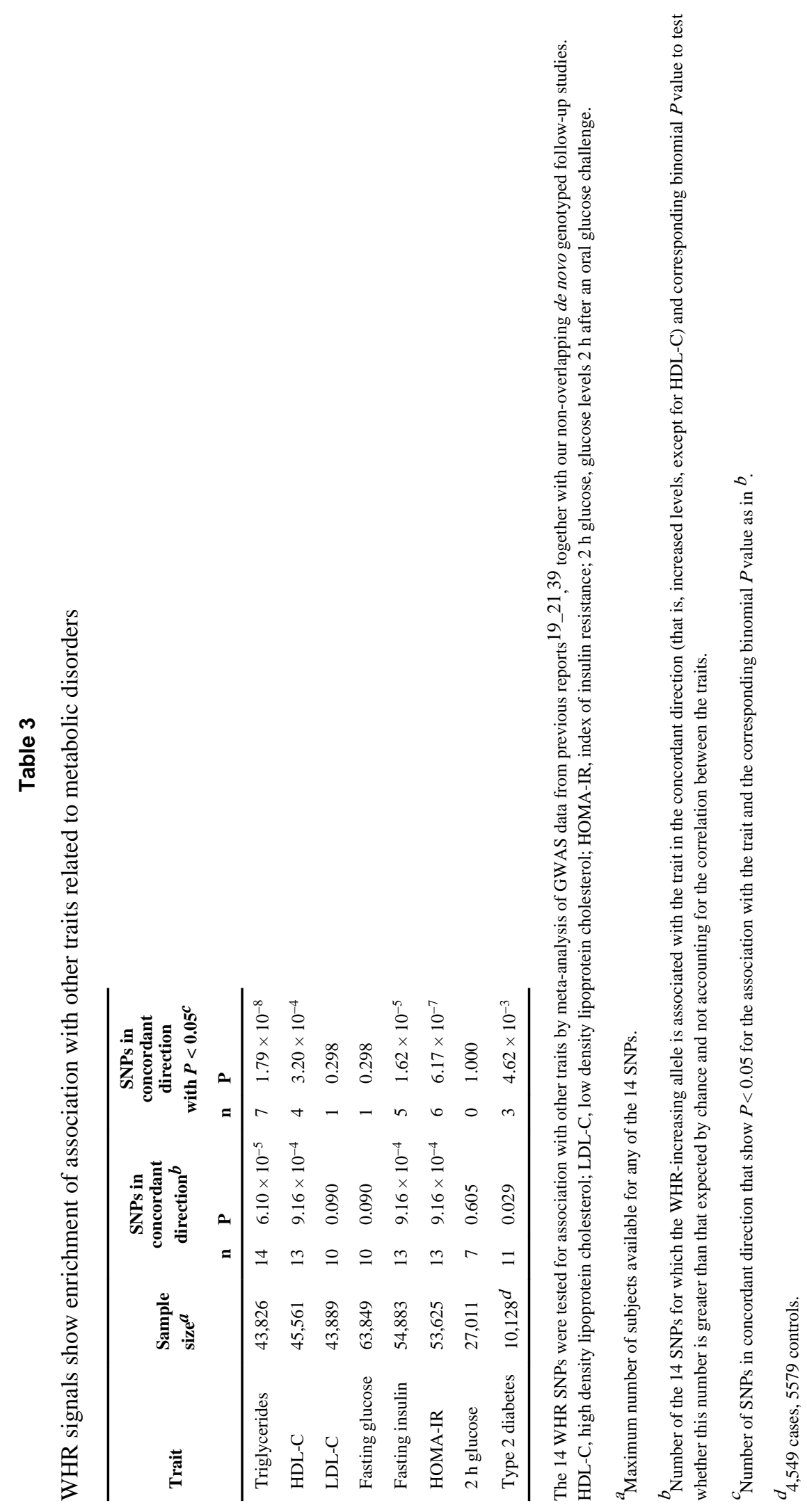




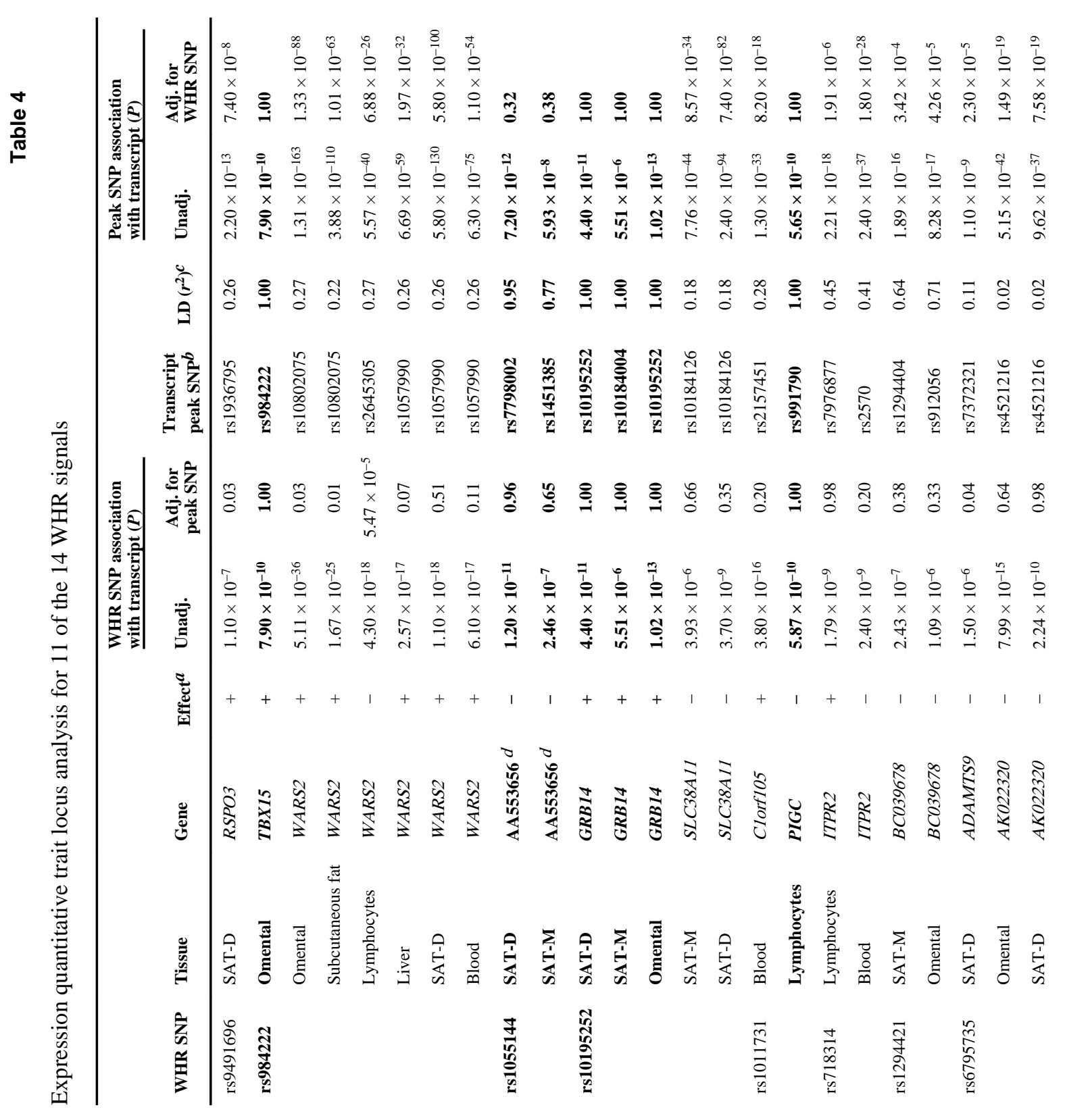




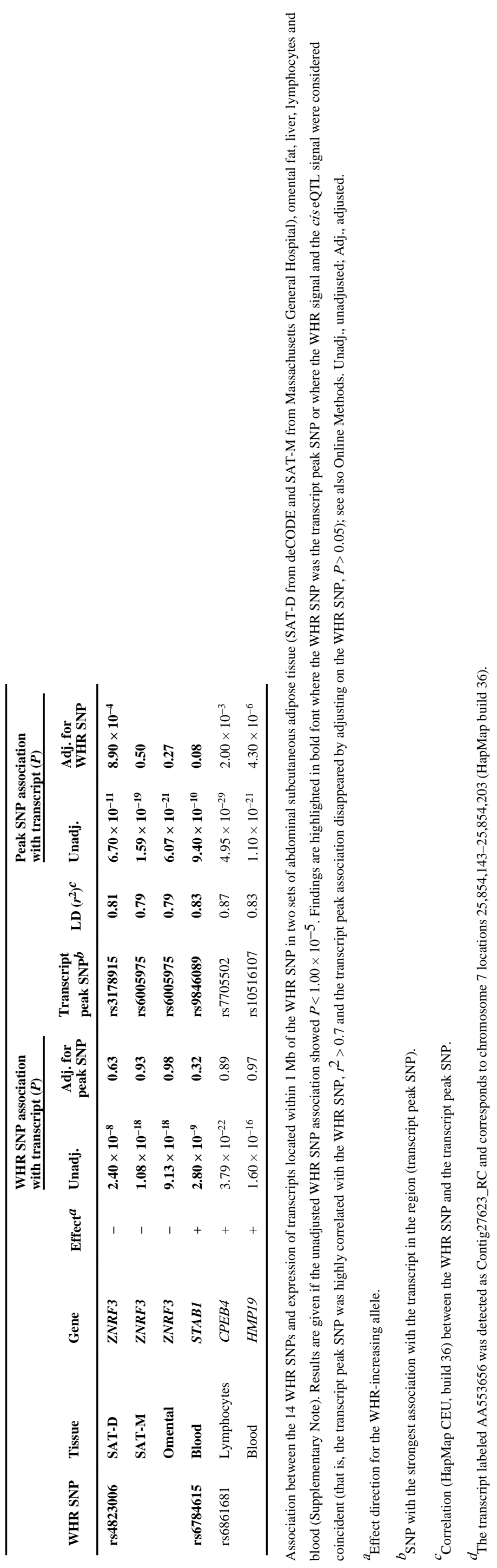

